\title{
Post-mortem histopathology underlying $\beta$ - amyloid PET imaging following flutemetamol F 18 injection
}

Milos D. Ikonomovic 1,7,13, Chris J. Buckley ${ }^{2}$, Kerstin Heurling 3,4 , Paul Sherwin ${ }^{5}$, Paul A. Jones ${ }^{2}$, Michelle Zanette ${ }^{5}$, Chester A. Mathis ${ }^{6}$, William E. Klunk ${ }^{7}$, Aruna Chakrabarty ${ }^{8}$, James Ironside ${ }^{9}$, Azzam Ismail ${ }^{8}$, Colin Smith ${ }^{10}$, Dietmar R. Thal ${ }^{11,14}$, Thomas G. Beach ${ }^{12}$, Gill Farrar² and Adrian P. L. Smith ${ }^{2 *}$

\begin{abstract}
In vivo imaging of fibrillar $\beta$-amyloid deposits may assist clinical diagnosis of Alzheimer's disease (AD), aid treatment selection for patients, assist clinical trials of therapeutic drugs through subject selection, and be used as an outcome measure. A recent phase III trial of $\left[{ }^{18} \mathrm{~F}\right]$ flutemetamol positron emission tomography (PET) imaging in 106 end-of-life subjects demonstrated the ability to identify fibrillar $\beta$-amyloid by comparing in vivo PET to post-mortem histopathology. Post-mortem analyses demonstrated a broad and continuous spectrum of $\beta$-amyloid pathology in $A D$ and other dementing and non-dementing disease groups. The GE067-026 trial demonstrated $91 \%$ sensitivity and $90 \%$ specificity of $\left[{ }^{18} \mathrm{~F}\right]$ flutemetamol PET by majority read for the presence of moderate or frequent plaques. The probability of an abnormal $\left[{ }^{18} \mathrm{~F}\right]$ flutemetamol scan increased with neocortical plaque density and AD diagnosis. All dementia cases with non-AD neurodegenerative diseases and those without histopathological features of $\beta$ amyloid deposits were $\left[{ }^{18} \mathrm{~F}\right]$ flutemetamol negative. Majority PET assessments accurately reflected the amyloid plaque burden in $90 \%$ of cases. However, ten cases demonstrated a mismatch between PET image interpretations and post-mortem findings. Although tracer retention was best associated with amyloid in neuritic plaques, amyloid in diffuse plaques and cerebral amyloid angiopathy best explain three $\left[{ }^{18} \mathrm{~F}\right]$ flutemetamol positive cases with mismatched (sparse) neuritic plaque burden. Advanced cortical atrophy was associated with the seven false negative $\left[{ }^{18} \mathrm{~F}\right]$ flutemetamol images. The interpretation of images from pathologically equivocal cases was associated with low reader confidence and inter-reader agreement. Our results support that amyloid in neuritic plaque burden is the primary form of $\beta$-amyloid pathology detectable with $\left[{ }^{18} \mathrm{~F}\right]$ flutemetamol PET imaging. ClinicalTrials.gov NCT01165554. Registered June 21, 2010; NCT02090855. Registered March 11, 2014.
\end{abstract}

Keywords: Flutemetamol, PET, Amyloid, Alzheimer's disease, Neuropathology (4-6 allowed)

\section{Introduction}

The relatively modest accuracy of a clinical diagnosis of Alzheimer's disease (AD) when compared to the definitive neuropathological findings at autopsy [4] demonstrates a current unmet need to detect the neuropathological hallmarks of $\mathrm{AD}$ ( $\beta$-amyloid plaques and neurofibrillary tangles) in life. Plaques containing fibrillar $\beta$-amyloid are readily detectable in histological tissue specimens using dyes with high affinity for amyloid $\beta$-sheet structure

\footnotetext{
*Correspondence: Adrian.Smith@ge.com

${ }^{2} \mathrm{GE}$ Healthcare, The Grove Centre (GC18), White Lion Road, Amersham,

Buckinghamshire HP7 9LL, UK

Full list of author information is available at the end of the article
}

(Thioflavin-S and Congo red). Analogues of these dyes have been radiolabelled to create positron emission tomography (PET) imaging tracers for detecting $\beta$ amyloid plaques in vivo. PET amyloid tracers such as Pittsburgh compound $\mathrm{B}\left(\left[{ }^{11} \mathrm{C}\right] \mathrm{PiB}\right)$ and its derivative $\left[{ }^{18} \mathrm{~F}\right]$ flutemetamol (Vizamyl ${ }^{\mathrm{TM}}$ ) generally can distinguish between the presence of moderate or frequent amyloid plaques, required for the diagnosis of $\mathrm{AD}$, or lesser densities that would rule out $\mathrm{AD}[24,61]$.

Results of recent studies using PET $\beta$-amyloid tracers have shown a good association between tracer retention and underlying $\beta$-amyloid plaques in brain autopsy and biopsy samples [11, 12, 28, 31, 35-38, 47, 50-52, 65-67]. 
However, many of these studies have drawn on a pool of end-of-life subjects with advanced disease, in which autopsy is anticipated shortly after PET imaging and with the specific goal to demonstrate efficacy. Furthermore these studies have been required to be measured against semiquantitative neuritic plaque counts as the historical standard of truth of amyloid burden. The criteria for subject selection in such studies is critical; selection of advanced dementia subjects and a group of subjects with preserved cognition will tend to give subject sets that are at the extreme ends of the $\beta$-amyloid spectrum (neuritic plaque densities of none or frequent by CERAD criteria [43]), biasing towards finding high test sensitivity and specificity. On the other hand, the likely beneficiaries of $\beta$-amyloid PET imaging would be those in early disease development with borderline $\beta$-amyloid pathology (sparse or moderate plaque densities by CERAD criteria). Such subjects may be most appropriate for trials testing disease-modifying drugs because they are more likely to respond to available treatments. Subjects with a limited life expectancy unrelated to cognitive status (e.g., elderly oncology patients) may have intermediate AD pathology, as well as preserved cognition.

In the recently reported $\left[{ }^{18} \mathrm{~F}\right]$ flutemetamol multicentre end-of-life $\beta$-amyloid imaging trials $[14,49]$, subjects were drawn from both dementia clinics and hospice centres. While visual PET image assessments were compared to neuritic plaque burden to demonstrate efficacy for regulatory approval, neuropathology diagnoses and multiregional histometric measures were also recorded including amyloid plaque assessment by immunohistochemistry and amyloid phase analysis. Rather than a dichotomous distribution consisting mainly of pathology extremes, the 106 subjects in this study represent a broad and continuous spectrum of $\beta$-amyloid pathology, as well as coincident neuropathology. This enabled assessment of subjects with cortical $\beta$-amyloid burdens that were borderline for clinicopathological significance, associated with diffuse or neuritic plaques as well as the effect of mixed pathology which is the subject of the present study.

\section{Materials and methods}

\section{Ethics, consent and permissions and consent to publish}

This was a phase 3 multicenter PET study of flutemetamol injection labelled with radioactive fluorine 18 $\left(\left[{ }^{18} \mathrm{~F}\right]\right.$ flutemetamol) for detecting brain $\mathrm{A} \beta$.

Institutional review boards or ethics committees at the following institutions approved the study protocol before initiation: St Margaret's Hospital and Moorgreen Hospital (Hammersmith, Queen Charlotte's \& Chelsea Research Ethics Committee); University of Michigan (The University of Michigan Medical School Institutional Review Board); Banner Alzheimer's Institute, Banner Sun
Health Research Institute, Premier Research Institute, Miami Jewish Health Systems, Galiz Research, VERITAS Research, LasVegas Radiology, Memory Enhancement Center and Compass Research (Western Institutional Review Board); Mt Sinai Medical Center, Wien Center for Alzheimer's Disease, (Mount Sinai Medical Center); Michigan State University (Michigan State University, Biomedical and Health Institutional Review Board); Barrows Neurological Institute (St. Joseph's Hospital \& Medical Center); Warren Alpert Medical School of Brown University (Lifespan Research Protection Office, RI, USA).

All participants or their legal representatives provided prior written informed consent including agreement to publish subject to anonymization.

\section{Trial subjects}

Subjects with cognitive status ranging from normal to advanced dementia and with a life expectancy of 12 months or less were enrolled at 16 centres in the United States and the United Kingdom. Of 180 enrolled subjects who were given $\left[{ }^{18} \mathrm{~F}\right]$ flutemetamol, 69 died within 13 months of imaging (Study GE067-007 [14]). Sixty-eight brains were evaluable for a) neuritic plaque assessment, b) correlation analysis between $\beta$-amyloid immunohistochemistry (IHC) and $\left[{ }^{18} \mathrm{~F}\right]$ flutemetamol standardised uptake value ratio (SUVR) values, and c) neuropathology diagnoses. Additional deaths occurring after this time period but prior to July 2013 allowed a further 39 evaluable brains to be included in a re-read trial (Study GE067-026). One subject in GE067-007, who died shortly after an accidental fall, underwent a Medical Examiner's autopsy, and this precluded regional mapping for the primary objectives. One GE067-026 brain was excluded as it was frozen rather than fixed at the site neuropathology laboratory. A total of 106 subjects were analysed in this study.

\section{Procedures \\ Tissue processing and sampling}

The whole brain was removed at autopsy, with brain stem and cerebellum attached, and the two cerebral hemispheres were separated. The entire left hemisphere was immersion fixed in $10 \%$ neutral buffered formalin for 21 days prior to transport. Dissection, sampling, macroscopic photography and examination, histological processing and staining were performed at a single central laboratory (Covance Laboratories Ltd, Harrogate UK) to minimize processing variability. Upon receipt of the fixed hemisphere, the brain stem and hindbrain were removed by transverse section of the midbrain at the level of the third cranial nerve, and the hemisphere was cut into $1-\mathrm{cm}$ coronal slices. Eight neocortical regions were sampled; the middle frontal gyrus, superior temporal gyrus, middle temporal gyrus, and inferior parietal 
lobe from the lateral cerebral surfaces (these regions are required by the Consortium to Establish a Registry for Alzheimer's Disease [CERAD] [43]) and the anterior cingulate gyrus, posterior cingulate gyri, precuneus, and primary visual cortex from the medial surface. Two 3-mm thick tissue blocks from the anterior and posterior surface of each 1-cm thick cortical sample were embedded in paraffin. Eleven additional paraffin tissue blocks were prepared to enable neuropathology diagnostics; i) olfactory tract and bulb, ii) striatum, iii) cholinergic basal forebrain nucleus, iv) thalamus, v) medulla, vi) pre- and post-central gyri, vii) anterior hippocampus, amygdala and entorhinal cortex, viii) hippocampal body, ix) pons, $\mathrm{x}$ ) midbrain, and xi) cerebellum. The neuropathology diagnostic slide set consisted of sections from the eight cortical regions and the eleven additional regions; nineteen regions in total. Histological analysis of the neocortical regions was restricted to the left hemisphere, as the right hemisphere was sometimes retained frozen for biochemical analysis and was not always available. Prior evidence indicated that $\beta$-amyloid pathology is typically symmetric in $\mathrm{AD}$ [44]. Three sections each separated by approximately $100 \mu \mathrm{m}$ were taken from both the anterior and posterior tissue blocks from each neocortical region.

\section{Neuritic plaque assessment}

The scoring of neuritic plaque density has been a standard part of AD neuropathology since 1991 Mirra et al. [33] and through two iterations of recommended guidelines Hyman et al. [26], Hyman et al. [25] and were therefore deemed the most appropriate measure as the standard of truth to demonstrate efficacy for market authorisation regulatory approval when the GE067-007 Clinical trial was initiated and for other amyloid PET tracers $[11,12,14]$. Indeed, the regulatory authorities required the neuritic plaques assessment as described by Mirra et al. [43]. However, it is recognised that neuritic plaques are identified by dystrophic neurites (Tauopathy) to which an amyloid tracer is not expected to bind and that amyloid PET tracers bind to the amyloid component in the neuritic plaques.

Bielschowsky silver staining [39] was pre-validated from standard techniques as the primary a priori histopathology standard of truth (SOT) for the presence of clinically significant cortical $\beta$-amyloid. The potential for inter-subject variation in staining was mitigated by centralising the staining at a single laboratory, including appropriate quality controls and recording staining quality on case report forms. For all subjects, Bielschowsky-stained slides were assessed semiquantitatively for neuritic plaque frequency in neocortical regions using published CERAD-based protocols; this methodology supports the subject-level rating for neuritic plaque density determined by the highest density (none, sparse, moderate or frequent) found in any 10x objective field and in any neocortical region examined [43]. While the use of this SOT may be suitable for supporting a diagnosis of $\mathrm{AD}$, other SOTs may be more suited for comparison to a more quantitative PET study. Accordingly, the classification of each case as normal or abnormal was determined by two additional methods (see Table 1), using multiple measures of neuritic plaque density in multiple regions. Multiple assessments mitigated focal heterogeneity that would be evident microscopically but not macroscopically at the lower $(6-\mathrm{mm})$ resolution of PET imaging. The 2 multi-measure methods were both modified from CERAD and differed mainly in the number of regions assessed. The first of these modified assessments averaged numeric assessments (arithmetic mean - see below) of neuritic plaque density in each of the 8 neocortical regions to give a continuous variable. This method was aligned to the global cortical PET assessment regions and formed our Standard of Truth $\left(\mathrm{mCERAD}_{\mathrm{SOT}}\right)$. The second method limited the assessment to only those regions originally recommended by CERAD and retained the categorical assignation of 'none, 'sparse,' 'moderate', or 'frequent' by employing the mode to average multiple categorical scores within regions (mCERAD mode $_{\text {) }}$, thus achieving a score more representative of the total area instead of being biased towards focal accumulations of plaques.

For the two modified CERAD assessments, a total of 30 measures per cortical region were recorded on case report forms by neuropathologists (JI and CS). Briefly, 5 randomly selected grey-matter fields of view per section were

Table 1 Bielschowsky assessments of neuritic plaque densities

\begin{tabular}{llll}
\hline Algorithm & mCERAD & mCET & $C E R A D$ \\
\hline Regions assessed & MFL, MTG, STG, IPL, ACG, PCG, PRC, PVC & MFL, MTG, STG, IPL & MFL, MTG, STG, IPL \\
Regional assessments & $\begin{array}{l}8 \text { regional averages (mean) of } 30 \\
\text { intra-regional numeric assessments } \\
\text { (each } 0 \leq x \leq 3 \text { ) }\end{array}$ & $\begin{array}{l}\text { 4 regional averages (mode) of } 30 \\
\text { intra-regional categorical assessments } \\
\text { (none, sparse, moderate, frequent) }\end{array}$ & $\begin{array}{l}1 \text { global assessment from region of } \\
\text { highest involvement (none, sparse, } \\
\text { moderate, frequent) }\end{array}$ \\
Case dichotomy & Any region $>1.5$ is abnormal & Any region = moderate or frequent is abnormal & moderate or frequent is abnormal
\end{tabular}

MFL midfrontal lobe, MTG middle temporal gyrus, STG superior temporal gyrus, IPL inferior parietal lobe, $A C G$ anterior cingulate gyrus, $P C G$ posterior cingulate gyrus, $P R C$ Precuneus, $P V C$ primary visual cortex

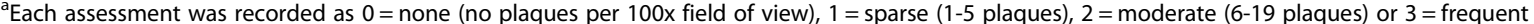

(20 or more plaques). A total of 240 cortical assessments were recorded for each case 
assessed and neuritic plaque densities were recorded as a score of $0=$ none (no plaques), $1=$ sparse ( $1-5$ plaques), $2=$ moderate $(6-19$ plaques), and $3=$ frequent (20+ plaques per 100x field of view [FoV]). [43, 60] For mCERAD ${ }_{\mathrm{SOT}}, 8$ regional scores were calculated as the arithmetic mean of the 30 measures per region. The scale midpoint was 1.5 , representing the threshold between sparse and moderate categories; a mean score $\leq 1.5$ was considered normal while a mean score of $>1.5$ was considered abnormal for each region. The dichotomous classification of the whole brain as $\beta$ amyloid normal or abnormal was aligned to the CERAD principle of the region of maximal involvement: if any one of the 8 regions was considered abnormal, i.e., any regional $\mathrm{mCERAD}_{\mathrm{SOT}}$ was $>1.5$, the whole brain was considered abnormal. Conversely, only if all regions were considered normal $(\leq 1.5)$ was the brain considered $\beta$-amyloid normal (see Table 1). The dichotomy of the brain as normal or abnormal was used for sensistivity and specificity calculations mandated as an endpoint for the trial whereas the continuous variable mCERAD $_{\mathrm{SOT}}$ provided a less discrete measure to compare to SUVR.

For $\mathrm{mCERAD}_{\text {mode }}$, the 30 measures made in each region (middle frontal, superior and middle temporal, and inferior parietal regions only) were averaged using mode to provide 4 regional categorical measures of none, sparse, moderate, or frequent. The brain was then dichotomised as abnormal if any region had a categorical assessment of moderate or frequent (see Table 1).

All measures were performed at a single laboratory to ensure consistency within the cohort as a consensus by two neuropathologists (JI and CS) against a priori criteria and blinded to clinical and imaging data and to the results of other histopathology analyses.

\section{$\beta$-amyloid immunohistochemistry}

Immunohistochemistry for $\beta$-amyloid was a secondary reference standard used for two sets of analyses (correlation with PET tracer retention measurements and neuropathology diagnoses). $\beta$-amyloid IHC (monoclonal antibody, clone 4G8; SIG-39220, Covance, USA, diluted 1:100) was performed on $5 \mu \mathrm{m}$ sections after $88 \%(\mathrm{v} / \mathrm{v})$ formic acid pre-treatment for $5 \mathrm{~min}$ and heat-mediated antigen retrieval and detected using biotinylated secondary antibody (DakoCytomation E0354, UK) and DABMap Kit (Ventana, USA). Staining was pre-validated and standardized on an automated Ventana Discovery XT staining module.

In the first 30 brains analysed, the percentage of greymatter area stained by the monoclonal antibody 4G8 was used for correlation analysis with regional PET SUVR measures. This analysis was performed on 3 step sections (separated by $100 \mu \mathrm{m}$ ) from the anterior block of each cortical region. For each of 5 randomly selected grey matter $100 x$ fields of view per section, automated histometric measures of the \% area of $\beta$-amyloid in 4G8stained sections were recorded. A mean value was determined for each cortical region. Stained sections were imaged using whole slide scanning (Aperio XT) with a pre-developed and validated macro (MATLAB; MathWorks Inc, MA, USA) used to threshold intensity, size, and morphometry after colour deconvolution to remove the haematoxylin staining channel. All histometric measures were performed at a single laboratory to ensure consistency within the cohort by individuals who were blinded to clinical and imaging data and to the results of other histopathology analyses.

\section{Neuropathology diagnoses}

For central laboratory neuropathological diagnosis, single sections from each of the 8 cortical regions and 11 additional neuropathology regions listed above were stained with haematoxylin and eosin, Bielschowsky silver (as above), $\beta$-amyloid IHC (as above), and Tau IHC (monoclonal antibody AT8; Cat No. MN1020, Thermo Scientific, UK). Additional sections from the entorhinal cortex and midbrain were stained for $\alpha$-synuclein (monoclonal antibody; NCL-L-ASYN, Leica Microsystems, U.K.) to assess Lewy body pathology, and ubiquitin (polyclonal antibody; Z0458, DakoCytomation, UK). Neuropathology reports were prepared, including macroscopic observations, microscopic findings and relevant interpretive diagnoses. Braak staging of neurofibrillary tangles (NFT) [8], CERAD scoring of neuritic plaque frequency [43], $\beta$ amyloid phase analysis [58], and AD likelihood according to the National Institute of Aging-Reagan Institute (NIARI) diagnostic criteria [26] were recorded on case report forms. Where additional analyses were reported by the referring sites (e.g. TDP-43 immunopositivity), these were transferred to the case report forms. Neuropathology diagnoses were made at a single centre by two neuropathologists ( $\mathrm{AC}$ and $\mathrm{AI}$ ) to ensure consistency across the cohort. A diagnosis of AD was made according to the National Institutes of Ageing - Reagan Institute criteria [9]. Both neuropathologists were blinded to clinical and imaging data and to the results of cortical neuritic plaque analyses. Because of blinding to clinical data, neuropathology diagnoses were made with the assumption of dementia. AD neuropathologic changes were also categorised post-hoc according to the more recent NIA-Alzheimer's Association criteria (NIA-AA) [25] using amyloid phase [58], Braak NFT stage and CERAD neuritic plaque score, collected above, as these criteria were published after trial initiation. Cerebral amyloid angiopathy (CAA) was categorised by Vonsattel grade, stage and type [57]. Other diagnoses were made against the following criteria; Tangle predominant dementia [68, 29], frontotemporal lobar 
degeneration (Picks Disease) [29], dementia with Lewy bodies [42], multiple system atrophy [22], and progressive supranuclear palsy $[23,40]$. It should be noted that subsequent to the trial additional guidelines and diagnostic criteria for AD [25] and primary age-related tauopathy [13] have been published.

\section{PET imaging and analysis}

The acquisition of $\left[{ }^{18} \mathrm{~F}\right]$ flutemetamol PET images and analysis are described in detail elsewhere [14]. Briefly, Flutemetamol F 18 Injection was administered intravenously at a dose of $185-370 \mathrm{MBq}$ of radioactivity at physician discretion, based on how long a scan the patient could be anticipated to tolerate; scan times were adjusted to dose to ensure equivalent scan quality with minimal patient discomfort. PET images were acquired in 2-min frames on PET/computerized tomography (CT) scanners, beginning approximately $90 \mathrm{~min}$ post injection. Typically, for an injected activity of $370 \mathrm{MBq}$, five 2-min frames were summed to give a 10 -min scan, which was attenuation-corrected using CT data. Most images were reconstructed iteratively to form axial slices of 128 X 128 pixels and with $~ 90$ slices covering the entire cerebrum. Image reconstructions were optimised at each PET centre using a NEMA IEC phantom [46] prior to patient imaging. In addition, a CT-scan was acquired. Equipment used to capture images varied across the study sites. The images were evaluated overall as either positive (abnormal/pathological) or negative (normal) for fibrillar $\beta$-amyloid in a blinded image evaluation (BIE) by 5 independent image readers who were blinded to all clinical, demographic, and pathology information. Readers were trained independently using GE Healthcare's automated reader training program [17, 54, 63]. The interactive training program gave instruction on brain anatomy, image display, and assessment methodology to classify 5 regions; frontal lobe, lateral temporal lobe, parietal lobe, posterior cingulate/precuneus, and the striatum as either normal or abnormal. Following the training, readers had to pass a test in which they had to correctly classify at least 14 out of 15 test cases as normal or abnormal. Five trained readers classified the study cases as normal or abnormal using the same reading and classifying methodology. If any one of the regions assessed in a given case image was deemed to be abnormal, the case was dichotomised as a positive image assessment [49]. The confidence with which a reader was able to reach the decision made was recorded on a scale of $1-5$ ( 5 being most confident). The majority read for each subject's PET image is defined as the image interpretation (normal/abnormal) made by at least 3 of the 5 readers. BIE analysis of the first 68 subjects (Study GE067-007) by 5 different readers from those used in GE067-026 are presented elsewhere [14] and are not part of this post-hoc analysis. It should be noted that PET assessments in this study was based solely upon image assessment by readers, and that SUVR measures (see below) were not used for primary dichotomous assessment as normal or abnormal.

\section{Mapping histopathology measures to quantitative PET image SUVR measures}

A detailed mapping regimen was implemented for the first 30 brains to co-register SUVR measurements with the 8 neocortical samples. SUVRs were measures of neocortical PET tracer retention normalised to the retention in the relatively spared cerebellum $\left(\mathrm{SUVR}_{\mathrm{cer}}\right)$ or pons $\left(\mathrm{SUVR}_{\mathrm{pon}}\right)$. Individual regional values were recorded and a composite SUVR (average across the 8 neocortical regions) was calculated. These measures were used as a pre-planned analysis to correlate tracer retention with $\beta$ amyloid histopathology as continuous rather than dichotomised variables. In addition to the first 30 brains, 2 further brains were added to this analysis post-hoc to understand the underlying pathology of false positives (cases 29 and 43 in Table 2). Photographs of the left hemispheres were taken pre- and post-sampling during the recording of macroscopic observations by the neuropathologist and during dissection of the fixed brains (Additional file 1). These included reconstructed hemispheres post-sampling to record the precise anatomical location from which the histological measures were made. These images were uploaded to the trial image repository and used to identify the regions sampled on ante-mortem anatomic images (CT or MRI) allowing registration with the $\mathrm{PET}$ images and enabling the relevant volumes of interest (VOIs) to be identified and to determine the regional SUVR. To ensure consistency across all subjects, this mapping was performed by a single individual $(\mathrm{KH})$ who was blinded to clinical data and histopathology.

\section{Data collection and analysis}

All data were collected from the clinical, imaging and pathology analysis centres via case report forms, which were quality-controlled before being transferred to a central data management centre (i3 Statprobe, Austin, Texas, USA, for trial GE067-007 and H2O Clinical LLC, Hunt Valley, Maryland, USA for GE067-026). Pathology data from the initial 68 brains in trial GE067-007 were used verbatim for the extended GE067-026 trial and no reanalysis was performed on these brains. All data blinds were maintained until the database was locked, at which point the data for the study primary objectives were analysed. The study GE067-026 [49] met its pre-defined success criteria of the lower bound of the $95 \%$ exact binomial confidence interval exceeding $75 \%$ for sensitivity and $60 \%$ for specificity for the majority interpretation. 
Table 2 Subject demographic information and data

\begin{tabular}{|c|c|c|c|c|c|c|c|c|c|c|c|c|c|}
\hline \multicolumn{5}{|c|}{ Demographics } & \multicolumn{7}{|c|}{ Neuropathology } & \multicolumn{2}{|c|}{ Imaging } \\
\hline Case & Age & Sex & Time $^{a}$ & Dementia $^{b}$ & $\overline{\mathrm{mCs} s^{c}}$ & $A \beta^{d}$ & $A D^{e}$ & CERAD $^{f}$ & Braak $^{9}$ & Amyloid phase ${ }^{h}$ & Diagnoses $^{i}$ & $\overline{\text { SUVR}^{j}}$ & PETmajk $^{k}$ \\
\hline 1 & 73 & $\mathrm{~F}$ & 360 & Yes & 0.0 & n.d. & Normal & - & $\|$ & 1 & Inf TDP' & 1.13 & Normal \\
\hline 2 & 84 & M & 17 & Yes & 0.0 & 0.3 & Normal & - & 1 & 0 & Normal & 1.18 & Normal \\
\hline 3 & 83 & M & 568 & No & 0.0 & n.d. & Normal & - & IV & 1 & LBD & 1.17 & Normal \\
\hline 4 & 91 & M & 130 & Yes & 0.0 & 0.9 & Normal & - & 0 & 0 & PSP VAD & 1.34 & Normal \\
\hline 5 & 63 & M & 433 & No & 0.0 & n.d. & Low & - & $\|$ & 1 & PSP & 1.42 & Normal \\
\hline 6 & 76 & $\mathrm{~F}$ & 145 & Yes & 0.0 & n.d. & Normal & - & $\|$ & 1 & LBD VAD & 1.22 & Normal \\
\hline 7 & 70 & M & 16 & No & 0.0 & n.d. & Normal & - & 0 & 0 & Normal & 1.44 & Normal \\
\hline 8 & 67 & M & 32 & No & 0.0 & n.d. & Normal & - & I & 0 & Normal & 1.37 & Normal \\
\hline 9 & 80 & M & 131 & Yes & 0.0 & n.d. & NA & - & III & 0 & TPD & 1.26 & Normal \\
\hline 10 & 61 & $\mathrm{~F}$ & 34 & No & 0.0 & n.d. & Normal & - & 0 & 1 & Normal & 1.67 & Normal \\
\hline 11 & 65 & $\mathrm{~F}$ & 393 & No & 0.0 & n.d. & Normal & - & III & 1 & $A C$ & 1.21 & Normal \\
\hline 12 & 60 & M & 374 & No & 0.0 & n.d. & Low & - & III & 1 & VAD & 1.34 & Normal \\
\hline 13 & 74 & M & 170 & Yes & 0.1 & 8.1 & Normal & - & 0 & 2 & VAD & 1.12 & Normal \\
\hline 14 & 66 & M & 155 & No & 0.1 & n.d. & Normal & - & 0 & 0 & $A C$ & 1.3 & Normal \\
\hline 15 & 76 & $\mathrm{~F}$ & 10 & Yes & 0.1 & 0.2 & Normal & - & I & 2 & Normal & 1.56 & Normal \\
\hline 16 & 63 & M & 12 & No & 0.1 & n.d. & Normal & - & 0 & 0 & Normal & 1.6 & Normal \\
\hline 17 & 73 & $\mathrm{~F}$ & 105 & Yes & 0.5 & 0.4 & Normal & - & V & 1 & TPD & 1.34 & Normal \\
\hline 18 & 90 & $\mathrm{~F}$ & 115 & Yes & 0.0 & n.d. & Low & S & III & 1 & $\operatorname{Inf} A S$ & 1.4 & Normal \\
\hline 19 & 89 & $\mathrm{~F}$ & 78 & No & 0.3 & n.d. & Int & S & IV & 2 & CAA AD & 1.25 & Normal \\
\hline 20 & 82 & $\mathrm{~F}$ & 24 & Yes & 0.4 & 0 & Low & S & III & 1 & LBD & 1.72 & Normal \\
\hline 21 & 92 & $\mathrm{~F}$ & 210 & Yes & 0.7 & n.d. & Normal & S & $\|$ & 4 & CAA PD & 1.55 & Normal \\
\hline 22 & 84 & $\mathrm{~F}$ & 69 & Yes & 1.1 & 1 & Int & S & $\|$ & 2 & $\operatorname{lnf}$ & 1.36 & Normal \\
\hline 23 & 72 & M & 142 & Yes & 1.3 & n.d. & Normal & S & 0 & 3 & FTD & 1.01 & Normal \\
\hline 24 & 87 & $\mathrm{~F}$ & 76 & Yes & 1.4 & 2 & Low & S & I & 3 & VAD & 1.57 & Normal \\
\hline 25 & 87 & $\mathrm{~F}$ & 137 & Yes & 1.5 & n.d. & Normal & S & 0 & 2 & AC AS & 1.53 & Normal \\
\hline 26 & 60 & M & 11 & Yes & 1.7 & n.d. & Low & S & $\|$ & 4 & CAA & 1.08 & Normal \\
\hline 27 & 81 & M & 189 & No & 1.8 & n.d. & Normal & $S$ & I & 4 & ND & 1.6 & Normal \\
\hline 28 & 92 & $\mathrm{~F}$ & 212 & Yes & 2.1 & n.d. & Int & S & III & 3 & TDP' & 1.26 & Normal \\
\hline 29 & 87 & $\mathrm{~F}$ & 131 & Yes & 1.4 & 8.1 & Low & S & IV & 5 & LBD & 1.95 & Abnormal \\
\hline 30 & 96 & $\mathrm{~F}$ & 630 & Yes & 1.9 & n.d. & High & S & $\mathrm{Vl}$ & 5 & $A D$ & 2.15 & Abnormal \\
\hline 31 & 92 & $\mathrm{~F}$ & 132 & Yes & 1.9 & n.d. & Low & S & III & 4 & LBD & 2.72 & Abnormal \\
\hline 32 & 89 & $\mathrm{~F}$ & 311 & Yes & 2.0 & n.d. & Int & S & III & 5 & AD CAA VAD & 2.33 & Abnormal \\
\hline 33 & 88 & $\mathrm{~F}$ & 118 & Yes & 2.1 & n.d. & Low & S & $\|$ & 4 & Inf LBD AS & 3.14 & Abnormal \\
\hline 34 & 80 & M & 2 & Yes & 2.1 & 7.6 & High & S & $\mathrm{Vl}$ & 5 & AD LBD & 2.1 & Abnormal \\
\hline 35 & 94 & $\mathrm{~F}$ & 19 & Yes & 2.1 & 7.7 & Int & S & III & 5 & $A D$ & 1.95 & Abnormal \\
\hline 36 & 88 & $\mathrm{~F}$ & 329 & Yes & 2.1 & n.d. & High & S & V & 5 & $A D$ & 2.84 & Abnormal \\
\hline 37 & 74 & $\mathrm{~F}$ & 550 & Yes & 2.8 & n.d. & High & S & $\mathrm{Vl}$ & 5 & $A D$ & 2.23 & Abnormal \\
\hline 38 & 86 & M & 19 & No & 1.4 & 2.3 & Int & M & III & 3 & $A D$ & 1.45 & Normal \\
\hline 39 & 75 & M & 64 & Yes & 1.4 & 1.4 & Int & M & $\|$ & 3 & $\operatorname{lnf} L B D$ & 1.23 & Normal \\
\hline 40 & 84 & M & 349 & Yes & 1.6 & n.d. & Int & M & V & 3 & AD LBD AS VAD Ath & 1.73 & Normal \\
\hline 41 & 93 & M & 323 & No & 1.9 & n.d. & Low & M & $\|$ & 3 & LBD & 1.36 & Normal \\
\hline 42 & 87 & M & 22 & No & 2.7 & 4 & Int & M & IV & 4 & $A D$ & 2.04 & Normal \\
\hline 43 & 86 & $\mathrm{~F}$ & 193 & Yes & 0.3 & 9.4 & Low & M & III & 4 & LBD & 2.07 & Abnormal \\
\hline
\end{tabular}


Table 2 Subject demographic information and data (Continued)

\begin{tabular}{|c|c|c|c|c|c|c|c|c|c|c|c|c|c|}
\hline \multicolumn{5}{|c|}{ Demographics } & \multicolumn{7}{|c|}{ Neuropathology } & \multicolumn{2}{|c|}{ Imaging } \\
\hline Case & Age & Sex & Time $^{a}$ & Dementia $^{b}$ & $\overline{m C s^{c}}$ & $A \beta^{d}$ & $A D^{e}$ & CERAD $^{f}$ & Braak $^{9}$ & Amyloid phase $^{h}$ & Diagnoses $^{i}$ & $\overline{\text { SUVR}^{j}}$ & PETmajk $^{k}$ \\
\hline 44 & 76 & $M$ & 84 & Yes & 1.5 & 10.3 & Low & $M$ & $\|$ & 3 & LBD & 1.87 & Abnormal \\
\hline 45 & 75 & M & 373 & Yes & 1.6 & n.d. & Int & M & IV & 5 & AD CAA & 1.48 & Abnormal \\
\hline 46 & 82 & $\mathrm{~F}$ & 127 & Yes & 1.7 & n.d. & Int & M & IV & 4 & $A D \perp B D$ & 2.32 & Abnormal \\
\hline 47 & 86 & M & 395 & Yes & 1.8 & n.d. & High & M & V & 5 & $A D$ & 2.83 & Abnormal \\
\hline 48 & 93 & $\mathrm{~F}$ & 500 & Yes & 1.8 & n.d. & Int & M & V & 3 & AD AS VAD & 1.6 & Abnormal \\
\hline 49 & 84 & M & 45 & Yes & 1.8 & n.d. & Low & M & $\|$ & 3 & VAD & 1.85 & Abnormal \\
\hline 50 & 93 & $\mathrm{~F}$ & 243 & Yes & 1.9 & n.d. & High & M & $\mathrm{Vl}$ & 5 & $A D$ & 2.72 & Abnormal \\
\hline 51 & 93 & $\mathrm{~F}$ & 755 & Yes & 2.0 & n.d. & High & M & IV & 5 & $A D$ & 2.2 & Abnormal \\
\hline 52 & 80 & M & 276 & Yes & 2.0 & n.d. & High & M & $\mathrm{VI}$ & 4 & $A D$ & 2.33 & Abnormal \\
\hline 53 & 90 & M & 308 & Yes & 2.1 & n.d. & Low & M & $\|$ & 4 & LBD & 2.19 & Abnormal \\
\hline 54 & 78 & M & 62 & Yes & 2.1 & 10.1 & High & M & $\mathrm{Vl}$ & 5 & $A D ~ L B D$ & 2.86 & Abnormal \\
\hline 55 & 86 & $\mathrm{~F}$ & 747 & Yes & 2.1 & n.d. & Int & M & IV & 3 & AD CAA & 1.81 & Abnormal \\
\hline 56 & 73 & $\mathrm{~F}$ & 295 & No & 2.2 & n.d. & Low & M & । & 3 & LBD & 1.76 & Abnormal \\
\hline 57 & 87 & $\mathrm{~F}$ & 318 & Yes & 2.2 & n.d. & Int & M & III & 5 & AD AS VAD Ath & 2.26 & Abnormal \\
\hline 58 & 88 & $\mathrm{~F}$ & 266 & Yes & 2.2 & n.d. & Int & M & III & 4 & $A D$ LBD & 1.91 & Abnormal \\
\hline 59 & 88 & $\mathrm{~F}$ & 79 & Yes & 2.3 & 17.6 & High & M & $\mathrm{Vl}$ & 5 & $A D$ & 1.67 & Abnormal \\
\hline 60 & 93 & $\mathrm{~F}$ & 396 & Yes & 2.3 & n.d. & High & M & $\mathrm{Vl}$ & 5 & AD CAA Inf & 3.08 & Abnormal \\
\hline 61 & 85 & M & 60 & No & 2.4 & 14.7 & High & M & $\mathrm{Vl}$ & 5 & CAA AD VAD & 2.81 & Abnormal \\
\hline 62 & 91 & M & 30 & Yes & 2.4 & 2.9 & High & M & V & 4 & $A D$ & 1.86 & Abnormal \\
\hline 63 & 95 & $\mathrm{~F}$ & 15 & Yes & 2.4 & 7.5 & High & M & $\mathrm{VI}$ & 5 & $A D$ & 1.89 & Abnormal \\
\hline 64 & 79 & M & 42 & No & 2.4 & n.d. & Int & M & III & 4 & CAA mCa AD & 2.44 & Abnormal \\
\hline 65 & 81 & $\mathrm{~F}$ & 184 & Yes & 2.5 & n.d. & Int & M & IV & 3 & LBD & 1.66 & Abnormal \\
\hline 66 & 84 & $\mathrm{~F}$ & 193 & Yes & 2.6 & n.d. & High & M & V & 5 & $A D$ & 2.4 & Abnormal \\
\hline 67 & 72 & M & 268 & Yes & 2.7 & n.d. & High & M & $\mathrm{Vl}$ & 5 & $A D \vee A D$ & 2.07 & Abnormal \\
\hline 68 & 63 & M & 342 & Yes & 2.8 & n.d. & High & M & $\mathrm{Vl}$ & 5 & AD AS VAD & 1.94 & Abnormal \\
\hline 69 & 89 & $\mathrm{~F}$ & 115 & Yes & 3.0 & n.d. & High & M & $\mathrm{Vl}$ & 5 & AD CAA LBD AS & 2.39 & Abnormal \\
\hline 70 & 83 & $\mathrm{~F}$ & 611 & Yes & 1.8 & n.d. & Low & $\mathrm{F}$ & । & 4 & AS CAA VAD TDP' & 1.87 & Normal \\
\hline 71 & 84 & $\mathrm{~F}$ & 189 & No & 1.7 & n.d. & Int & $\mathrm{F}$ & 0 & 3 & CAA Inf VAD AD & 2.02 & Abnormal \\
\hline 72 & 82 & M & 397 & Yes & 1.9 & n.d. & High & $\mathrm{F}$ & $\mathrm{VI}$ & 5 & AD CAA VAD & 2.41 & Abnormal \\
\hline 73 & 86 & $\mathrm{~F}$ & 155 & Yes & 2.0 & n.d. & High & $\mathrm{F}$ & V & 5 & $A D$ & 2.43 & Abnormal \\
\hline 74 & 93 & $\mathrm{~F}$ & 594 & Yes & 2.0 & n.d. & High & $\mathrm{F}$ & IV & 5 & $A D$ & 2.46 & Abnormal \\
\hline 75 & 90 & $\mathrm{~F}$ & 538 & Yes & 2.0 & n.d. & High & $\mathrm{F}$ & $\mathrm{Vl}$ & 4 & AD CAA AS VAD & 2.78 & Abnormal \\
\hline 76 & 78 & $\mathrm{~F}$ & 180 & Yes & 2.2 & n.d. & Normal & $\mathrm{F}$ & 0 & 4 & MSA & 2.03 & Abnormal \\
\hline 77 & 93 & $\mathrm{~F}$ & 200 & Yes & 2.2 & n.d. & High & $\mathrm{F}$ & V & 5 & AD AS CAA LBD VAD & 2.42 & Abnormal \\
\hline 78 & 78 & $\mathrm{~F}$ & 125 & Yes & 2.2 & n.d. & High & $\mathrm{F}$ & $\mathrm{Vl}$ & 5 & AD LBD & 2.12 & Abnormal \\
\hline 79 & 72 & M & 1 & Yes & 2.4 & 11.4 & High & $\mathrm{F}$ & $\mathrm{Vl}$ & 5 & $A D$ & 2.37 & Abnormal \\
\hline 80 & 76 & $\mathrm{~F}$ & 27 & Yes & 2.4 & n.d. & High & $\mathrm{F}$ & $\mathrm{Vl}$ & 5 & AD CAA & 1.83 & Abnormal \\
\hline 81 & 77 & $\mathrm{~F}$ & 11 & Yes & 2.4 & 8.9 & High & $\mathrm{F}$ & $\mathrm{Vl}$ & 4 & $A D$ & 1.59 & Abnormal \\
\hline 82 & 91 & $\mathrm{~F}$ & 55 & Yes & 2.4 & 11.2 & High & $\mathrm{F}$ & $\mathrm{VI}$ & 4 & AD CAA & 2.2 & Abnormal \\
\hline 83 & 81 & M & 204 & Yes & 2.4 & n.d. & High & $\mathrm{F}$ & $\mathrm{Vl}$ & 4 & AD CAA LBD & 2.41 & Abnormal \\
\hline 84 & 82 & M & 15 & Yes & 2.5 & 6.9 & High & $\mathrm{F}$ & $\mathrm{Vl}$ & 4 & AD CAA & 2.23 & Abnormal \\
\hline 85 & 83 & M & 34 & Yes & 2.5 & 8.5 & High & $\mathrm{F}$ & $\mathrm{Vl}$ & 5 & $A D$ & 2.6 & Abnormal \\
\hline 86 & 90 & $\mathrm{~F}$ & 51 & Yes & 2.5 & 12.2 & High & $\mathrm{F}$ & $\mathrm{VI}$ & 4 & $A D$ & 2.35 & Abnormal \\
\hline
\end{tabular}


Table 2 Subject demographic information and data (Continued)

\begin{tabular}{|c|c|c|c|c|c|c|c|c|c|c|c|c|c|}
\hline \multicolumn{5}{|c|}{ Demographics } & \multicolumn{7}{|c|}{ Neuropathology } & \multicolumn{2}{|c|}{ Imaging } \\
\hline Case & Age & Sex & Time $^{a}$ & Dementia $^{b}$ & $\overline{\mathrm{mCs} s^{c}}$ & $A \beta^{d}$ & $A D^{e}$ & CERAD $^{f}$ & Braak $^{9}$ & Amyloid phase ${ }^{h}$ & Diagnoses $^{i}$ & $\overline{\text { SUVR}^{j}}$ & PETmaj ${ }^{k}$ \\
\hline 87 & 73 & $F$ & 27 & Yes & 2.5 & 9.6 & High & $F$ & $\mathrm{Vl}$ & 5 & $A D$ & 2.23 & Abnormal \\
\hline 88 & 87 & M & 1 & Yes & 2.5 & 7.8 & High & $\mathrm{F}$ & IV & 4 & AD CAA & 2.1 & Abnormal \\
\hline 89 & 89 & $\mathrm{~F}$ & 768 & Yes & 2.5 & n.d. & High & $F$ & V & 5 & AD CAA LBD AS & 1.93 & Abnormal \\
\hline 90 & 79 & M & 332 & Yes & 2.5 & n.d. & High & $\mathrm{F}$ & $\mathrm{Vl}$ & 5 & AD CAA LBD & 2.24 & Abnormal \\
\hline 91 & 80 & M & 0 & Yes & 2.6 & 7.9 & High & F & $\mathrm{Vl}$ & 5 & $A D$ & 2 & Abnormal \\
\hline 92 & 79 & $\mathrm{~F}$ & 422 & Yes & 2.6 & n.d. & High & $\mathrm{F}$ & V & 5 & AD CAA LBD AS VAD HC & 2.38 & Abnormal \\
\hline 93 & 87 & M & 106 & Yes & 2.6 & n.d. & High & F & $\mathrm{Vl}$ & 5 & $A D$ & 2.2 & Abnormal \\
\hline 94 & 66 & $\mathrm{~F}$ & 139 & Yes & 2.7 & n.d. & High & $\mathrm{F}$ & $\mathrm{Vl}$ & 5 & $A D$ & 2.37 & Abnormal \\
\hline 95 & 84 & M & 181 & Yes & 2.7 & n.d. & High & $\mathrm{F}$ & V & 4 & $A D$ LBD & 2.75 & Abnormal \\
\hline 96 & 87 & M & 769 & Yes & 2.7 & n.d. & High & $\mathrm{F}$ & $\mathrm{Vl}$ & 5 & AD CAA LBD VAD & 2.5 & Abnormal \\
\hline 97 & 71 & M & 305 & Yes & 2.7 & n.d. & High & $\mathrm{F}$ & V & 5 & AD CAA & 2.47 & Abnormal \\
\hline 98 & 72 & $\mathrm{~F}$ & 565 & Yes & 2.7 & n.d. & High & $\mathrm{F}$ & $\mathrm{Vl}$ & 5 & AD CAA LBD & 2.58 & Abnormal \\
\hline 99 & 85 & M & 846 & Yes & 2.8 & n.d. & High & F & $\mathrm{Vl}$ & 5 & $A D \vee A D$ & 2.01 & Abnormal \\
\hline 100 & 84 & $\mathrm{~F}$ & 198 & Yes & 2.8 & 6.3 & High & F & $\mathrm{Vl}$ & 4 & $A D$ & 1.48 & Abnormal \\
\hline 101 & 85 & $\mathrm{~F}$ & 436 & Yes & 2.9 & n.d. & High & $\mathrm{F}$ & $\mathrm{Vl}$ & 5 & $A D$ & 2.65 & Abnormal \\
\hline 102 & 75 & $\mathrm{~F}$ & 66 & Yes & 2.9 & 10.6 & High & F & $\mathrm{Vl}$ & 5 & $A D$ & 2.47 & Abnormal \\
\hline 103 & 87 & M & 493 & No & 2.9 & n.d. & High & F & IV & 5 & CAA LBD AD & 2.42 & Abnormal \\
\hline 104 & 86 & $\mathrm{~F}$ & 127 & Yes & 3.0 & n.d. & High & F & $\mathrm{Vl}$ & 5 & $A D$ & 2.9 & Abnormal \\
\hline 105 & 81 & M & 171 & Yes & 3.0 & n.d. & High & $\mathrm{F}$ & $\mathrm{Vl}$ & 5 & $A D$ & 2.34 & Abnormal \\
\hline 106 & 63 & M & 562 & Yes & 3.0 & n.d. & High & F & $\mathrm{Vl}$ & 5 & $A D$ & 2.72 & Abnormal \\
\hline
\end{tabular}

Subjects are ranked by CERAD neuritic plaque frequency and then by mCERAD SOT. $A C$ ageing changes, $A D$ Alzheimer's disease (high or intermediate likelihood by National Institute of Ageing-Reagan Institute criteria), AS arteriosclerosis or arteriolosclerosis, Ath atherosclerosis, CAA cerebral amyloid angiopathy, FTD frontotemporal lobar degeneration, HC hydrocephalus, Inf infarct, $L B D$ Lewy body disease, $m C a$ metastatic carcinoma, MSA multisystem atrophy, ND neurofibrillary degeneration, PD Parkinson's disease, PSP progressive supranuclear palsy, SUVR standard retention value ratio, TDP+ TDP43 immunopositivity, TPD tangle-predominant dementia, VSD vascular disease not otherwise specified

aTime between PET imaging and death in days

${ }^{b}$ Dementia recorded in the study medical history

${ }^{\prime}$ mCERAD $D_{\text {SOT; }}$ maximal regional mean score determining Standard of Truth assignation as abnormal if $>1.5$

dPercentage area of grey matter stained positively by amyloid $\beta$ immunohistochemistry (4G8) determined only on a subset ( 32 subjects) of the cohort eAlzheimer's Disease likelihood recorded by a neuropathologist against National Institute of Ageing-Reagan Institute criteria [26] but blinded to dementia status ${ }^{f}$ CERAD neuritic plaque frequency recorded during neuropathology diagnoses (N: none; S: sparse; M: moderate; F: frequent)

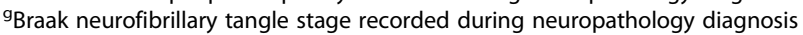

hamyloid phase $[25,58]$

'Neuropathologist's diagnoses blinded to clinical data. Note: co-incident plaque burden may be present in non-AD diagnoses

jComposite SUVR determined from bilateral measures and normalised to cerebellum as the reference region

${ }^{\mathrm{k}}$ Majority PET image evaluation

'TDP43 immunopositivity was recorded at the site neuropathology laboratories, not as part of the diagnoses for the GE studies. This analysis was not performed on all subjects

All analyses presented here represent post-hoc analyses of pathology data collected during the clinical trials GE067-007 $(N=68)$ and GE067-026 $(N=106)$, regional co-registered histopathology correlates and SUVRs collected in a subset $(N=32)$ of the GE067-007 subjects, and PET image evaluation by 5 independent readers of all 106 subjects read as a single trial GE067-026.

Receiver operating characteristic (ROC) analysis was used to determine optimal threshold values for the post-hoc analysis of regional mCERAD ${ }_{\mathrm{SOT}}, \beta$-amyloid $\mathrm{IHC} \%$ area measures, and for SUVR thresholds. SUVR comparisons were performed using parametric analysis of variance (ANOVA). The Fleiss coefficient $P_{i}$ was used to measure inter-reader agreement $\left[P_{i}=1 / n(n-1)^{*}\left(A^{2}+N^{2}-n\right)\right.$, where $n$ is the number of readers, $\mathrm{A}$ is the number calling the case abnormal, and $\mathrm{N}$ is the number of readers calling the case negative] [18]. Non-parametric tests for correlation (Spearman's), differences (Wilcoxon Rank Sum Test or MannWhitney $U$ test, Kruskal-Wallis test), and contingency analysis ( $\chi^{2}$ and Fisher's Exact Test) were used for all other analyses where indicated. All statistical tests were performed using StatSoft Statistica software (Tulsa, USA) unless otherwise stated. All graphs and figures (Except Figs. 1 and 3) were also produced using Statistica software. Graphical representation in box plots represent mean +/- 1 standard error (SE, boxes) and 95\% confidence intervals 


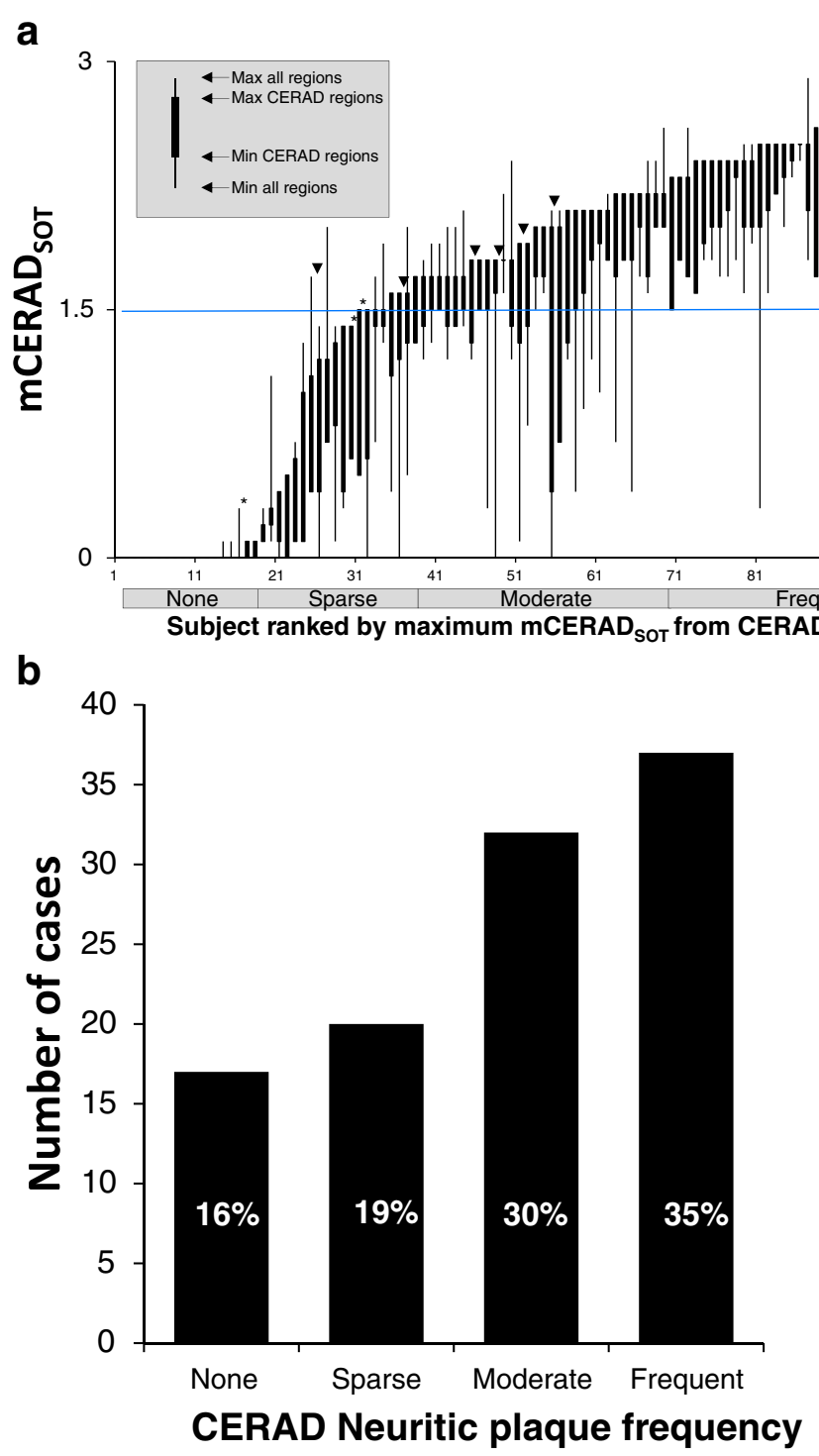

Fig. 1 The GE067-026 cohort contained a broad and continuous spectrum of neocortical neuritic plaque burden. Panel a. Subjects ranked by the maximal mCERAD SOT score for CERAD regions demonstrating a number of cases where dichotomy was based on a burden close to the threshold of 1.5. (cases were determined to be abnormal if any regional score was greater than 1.5, i.e. if the upper 'whiskers' in the plot are over 1.5). Most disparities between pathology and PET dichotomy as abnormal or normal occur in cases where the neuritic plaque mCERAD the threshold. Downward arrowheads indicate abnormal cases that were assessed as normal by PET (false negatives) and asterisks indicate normal cases assessed as abnormal (false positives). Panel $\mathbf{b}$. The spread of cases categorised by CERAD neuritic plaque frequency

(whiskers). Outlier and extreme values are presented as open circles and asterisks, respectively and are identified by the Statistica software as; outlier values > UBV + o.c." (UBVLBV) $\mathrm{OR}<\mathrm{LBV}$ - o.c." (UBV • LBV); extreme values > UBV $+2 *$ o.c.* (UBV-LBV) OR < LBV - 2* o.c.* (UBV • LBV) where $\mathrm{UBV}$ is the upper box value (mean $+1 \mathrm{SE}$ ), $\mathrm{LBV}$ is the lower box value (mean-1SE) and o.c. is the outlier coefficient set at 1.5. Note for probability plots (Figs. 2a-d, 6c and 7b, d \& e) all values are either 0 or 1 and extreme and outlier values will always be 0 or 1 . For confidence plots (Figs. $4 \mathrm{c}$ and $5 \mathrm{~b}$ ) all values are integers between 1 and 5 and so all outlier and extreme values will be integers. For inter-reader agreement plots (Figs. $4 \mathrm{a} \& \mathrm{~b}$ and $5 \mathrm{a}) \mathrm{P}_{\mathrm{i}}$ values are 1 (5/5 agreement), 0.6 (4:1 or 1:4 agreement) or 0.4 (3:2 or $2: 3$ agreement) and all outlier and extreme values will have these values. For all other plots outliers and extremes are continuous variables.

\section{Results}

The GE067-026 cohort of 106 brains included a broad and continuous spectrum of $\beta$-amyloid pathology based on mCERAD SOT $_{\text {SOT }}$ aERAD assessments (Fig. 1a). Seventy-six brains (72\%) were determined to be 

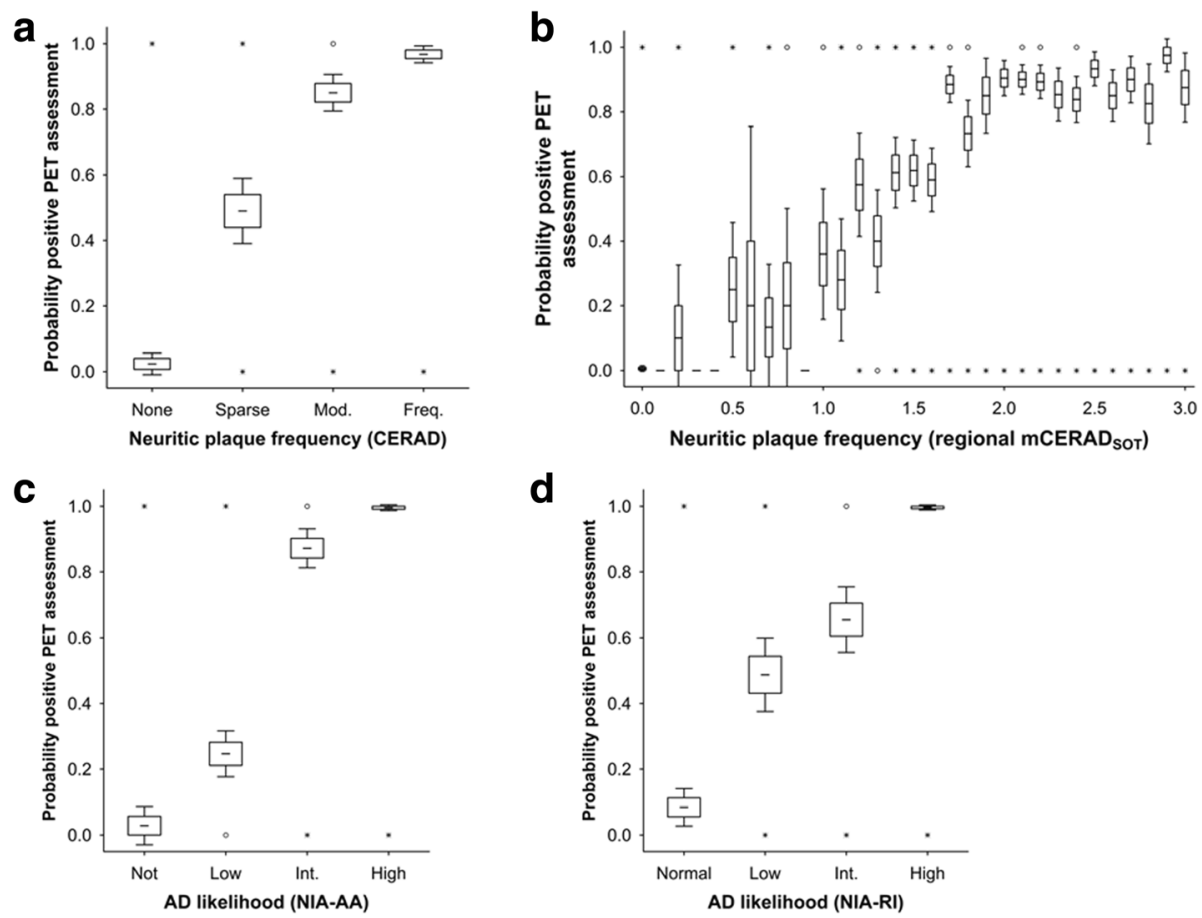

Fig. 2 The probability that the PET image is interpreted as positive increases with cortical neuritic plaque burden. Panel a. The probability (0-1) of a PET positive assessment increases with CERAD neuritic plaque frequency $(N=106)$. Panel $\mathbf{b}$. The probability of PET positive image assessment by neocortical regional mCERAD $\mathrm{SOT}$ score $(N=424,4$ neocortical regions per case; frontal, temporal, parietal and posterior cingulate/precuneus). Panel $\mathbf{c} \& \mathbf{d}$. The probability of PET positive image assessment increases by AD diagnosis against the NIA-AA (Panel $\mathbf{c}$ ) and NIA-RI criteria (Panel $\mathbf{d}$ ). For all panels, boxes represent mean $+/-1$ standard error and whiskers represent 95\% confidence interval. Open circles represent outlier values and asterisks represent extreme values (see Materials and methods for details)

abnormal by mCERAD ${ }_{\mathrm{SOT}}$, and $30(28 \%)$ were assessed as normal; together, these brains provided a continuous distribution of cases throughout the range of neuritic plaque pathology (Fig. 1b). Demographic data, pathology, and majority PET BIE assessments for all subjects are given in Table 2. Sixty-six cases had a neuropathological diagnosis of $\mathrm{AD}(62 \%)$, defined as either 'intermediate' or 'high' likelihood by NIA-RI criteria [26], in which neuritic plaques are nominally moderate or frequent in the neocortex. While $\mathrm{AD}$ was the predominant neuropathological diagnosis (Table 3), it was often recorded with coincident pathologies, such as CAA $(n=24)$ or other vascular disease $(n=27)$, or Lewy body disease (LBD, $n=16$ ). Just less than half of the $66 \mathrm{AD}$ cases had "pure" AD $(n=30)$. Lewy body disease was the next most predominant diagnosis $(n=$ 29 ; $27 \%$ ) and, in addition to the 16 cases with coincident $A D$ pathology sufficient for a diagnosis of $A D$, many other cases had varying degrees of co-incident $\beta$-amyloid neuropathology (Table 2). Indeed, many of the subjects without a primary diagnosis of $\mathrm{AD}$ had a range of coincident $\mathrm{AD}$ pathology, potentially allowing assessment of PET imaging in a target population where underlying $\mathrm{AD}$ pathology may be coincident with other dementing disorders. This mixed pathology population included cases with neuritic plaque frequencies close to the dichotomous threshold, i.e. between sparse (normal) and moderate (abnormal) which was the presumed a priori lower limit of plaque burden detectable with $\left[{ }^{18} \mathrm{~F}\right]$ flutemetamol.

Subjects classified as abnormal by mCERAD ${ }_{\text {SOT }}$ were generally older than those categorised as normal $(P<$ 0.01 , Mann-Whitney) and the average age of female subjects was slightly older than that of males (81.5 vs 78.5 years respectively; $P<0.001$ Mann-Whitney). mCERAD $_{\text {SOT }}$ was independent of gender $(P=0.57$ Mann-Whitney). Most histometric measures of plaques, tangles, and diagnoses were correlated (Table 4), as would be expected, given that the diagnoses of $A D$ were derived from these measures.

Time between PET scan and death did not significantly influence the outcome although we note that one false negative case died 611 days after PET. While it is possible that the amyloid burden increased during this period we are unable to confirm this.

\section{Blinded image evaluations of PET Images}

Seventy-two cases were assessed as positive (abnormal) by majority read and the remaining 34 cases were normal by majority read (Table 2). As $\beta$-amyloid 
Table 3 PET majority, AD and mCERADsot assessment by disease category

\begin{tabular}{|c|c|c|c|c|c|}
\hline Disease category & Number & Percent & PETmaj abnormal & \# With coincident $A D^{a}$ & \# mCERAD ${ }_{\text {SOT }}$ abnormal \\
\hline Alzheimer's Disease ${ }^{a}$ & 66 & $(62 \%)$ & 62 & 66 & 64 \\
\hline Lewy Body Disease ${ }^{b}$ & 29 & $(27 \%)$ & 23 & 16 & 22 \\
\hline Cerebral Amyloid Angiopathy ${ }^{c}$ & 27 & $(25 \%)$ & 23 & 24 & 25 \\
\hline Vascular ${ }^{d}$ & 21 & $(20 \%)$ & 14 & 14 & 16 \\
\hline Arteriosclerosis $^{\mathrm{e}}$ & 13 & $(12 \%)$ & 9 & 9 & 11 \\
\hline Infarct & 7 & $(7 \%)$ & 3 & 2 & 3 \\
\hline Normal & 6 & $(6 \%)$ & 0 & 0 & 0 \\
\hline Ageing Changes & 3 & $(3 \%)$ & 0 & 0 & 0 \\
\hline TDP43 immunopositive ${ }^{f}$ & 3 & $(3 \%)$ & 0 & 0 & 2 \\
\hline Tangle predominant dementia & 2 & $(2 \%)$ & 0 & 0 & 0 \\
\hline Progressive Supranuclear Palsy & 2 & $(2 \%)$ & 0 & 0 & 0 \\
\hline Atherosclerosis & 2 & $(2 \%)$ & 1 & 2 & 2 \\
\hline Frontotemporal Dementia & 1 & $(1 \%)$ & 0 & 0 & 0 \\
\hline Metastatic carcinoma & 1 & $(1 \%)$ & 1 & 1 & 1 \\
\hline Hydrocephalus & 1 & $(1 \%)$ & 1 & 1 & 1 \\
\hline Multiple system atrophy & 1 & $(1 \%)$ & 1 & 0 & 1 \\
\hline Neurofibrillary degeneration & 1 & $(1 \%)$ & 0 & 0 & 1 \\
\hline Parkinson's Disease & 1 & $(1 \%)$ & 0 & 0 & 0 \\
\hline
\end{tabular}

$A D$ Alzheimer's disease, $m C E R A D_{S O T}$ standard of truth by modified Consortium to Establish a Registry for Alzheimer's Disease criteria, $N$ Number of subjects, PETmaj majority read of positron emission tomography images, SOT standard of truth

All neuropathology diagnoses made blind to clinical data

ancludes intermediate or high likelihood by National Institute of Ageing-Reagan Institute criteria irrespective of dementia status

${ }^{b}$ Diagnosed as dementia with Lewy bodies blinded to clinical data

'Does not include focal cerebral amyloid angiopathy

Includes: multifocal infarcts, microinfarcts, cerebral vascular disease, vascular brain injury and vascular dementia

eincludes arteriosclerosis and arteriolosclerosis

${ }^{\mathrm{f}}$ TDP43 immunopositivity was recorded at the site neuropathology laboratories, not as part of the diagnoses for the GE studies. This analysis was not performed on all subjects

is believed to deposit in phases, starting with the neocortex and advancing to subcortical regions [58], the pattern of regional PET assessment was examined. Only one case was classified as abnormal on the basis of a single cortical region showing abnormal retention (case 49). All other cases assessed as positive by majority were positive in more than one neocortical region. With respect to cortical versus striatal results (with the potential to compare to $\beta$-amyloid phases [58]), 6 cases were cortical positive and striatal negative (cases 44, 48, 49, 55, 58 and 64) and no case was deemed majority positive solely on the basis of an abnormal striatal retention pattern. Of the 72 majority BIE positive cases, $55(76 \%)$ were abnormal in all 5 regions assessed and thus clearly positive. The posterior cingulate/precuneus was deemed abnormal in all cases positive by majority BIE. The striatum was deemed abnormal in 66 of these 72 positive cases by majority BIE.

The probability of the PET image being rated as abnormal increased with neocortical neuritic plaque frequency and $\mathrm{AD}$ diagnosis (Fig. 2). Illustrations of PET images alongside representative histopathology for select case are shown in Fig. 3, including some cases with discordant pathology versus PET results, which are discussed later.

Reader confidence was higher in cases deemed abnormal by mCERAD ${ }_{\text {SOT }}$ than in those deemed $\beta$ amyloid normal ( $P=0.000125$, Mann Whitney $U$ test), suggesting that readers felt more confident identifying abnormal retention patterns than the normal whitematter retention pattern. Unsurprisingly, reader confidence was highest in highly abnormal cases i.e. CERAD frequent or amyloid phase 5 cases (Fig. 4) and interreader agreement was highest in cases that were either clearly positive (phase $5 /$ frequent) or negative (phase $0-2 /$ none). When neocortical $\beta$-amyloid pathology was close to the threshold (sparse/moderate or amyloid phase 3) reader confidence and inter-reader agreement worsened (Fig. 4). Readers were less confident assessing cases that were subsequently classified as false negatives compared to true negatives or true positives $(P<$ 0.05 and $P<0.001$ respectively Mann-Whitney $U$ test) and inter-reader agreement was also worse (Fig. $5 ; P<$ 


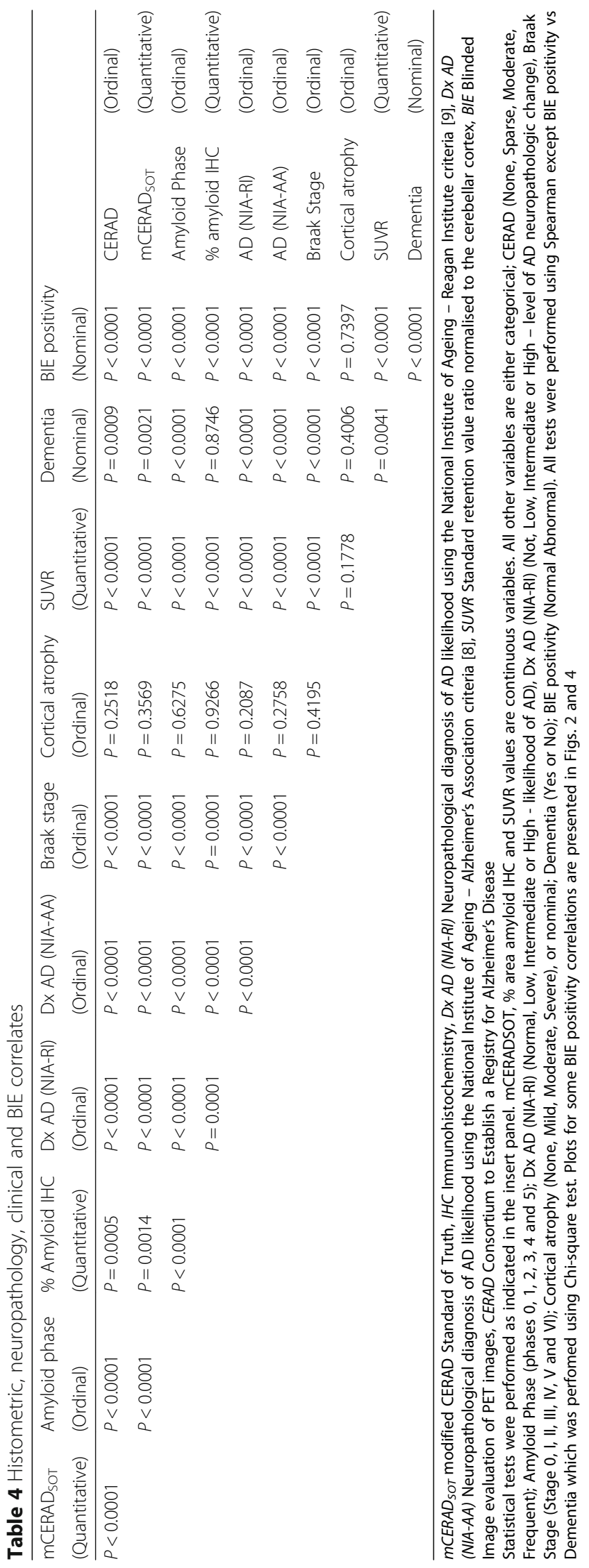




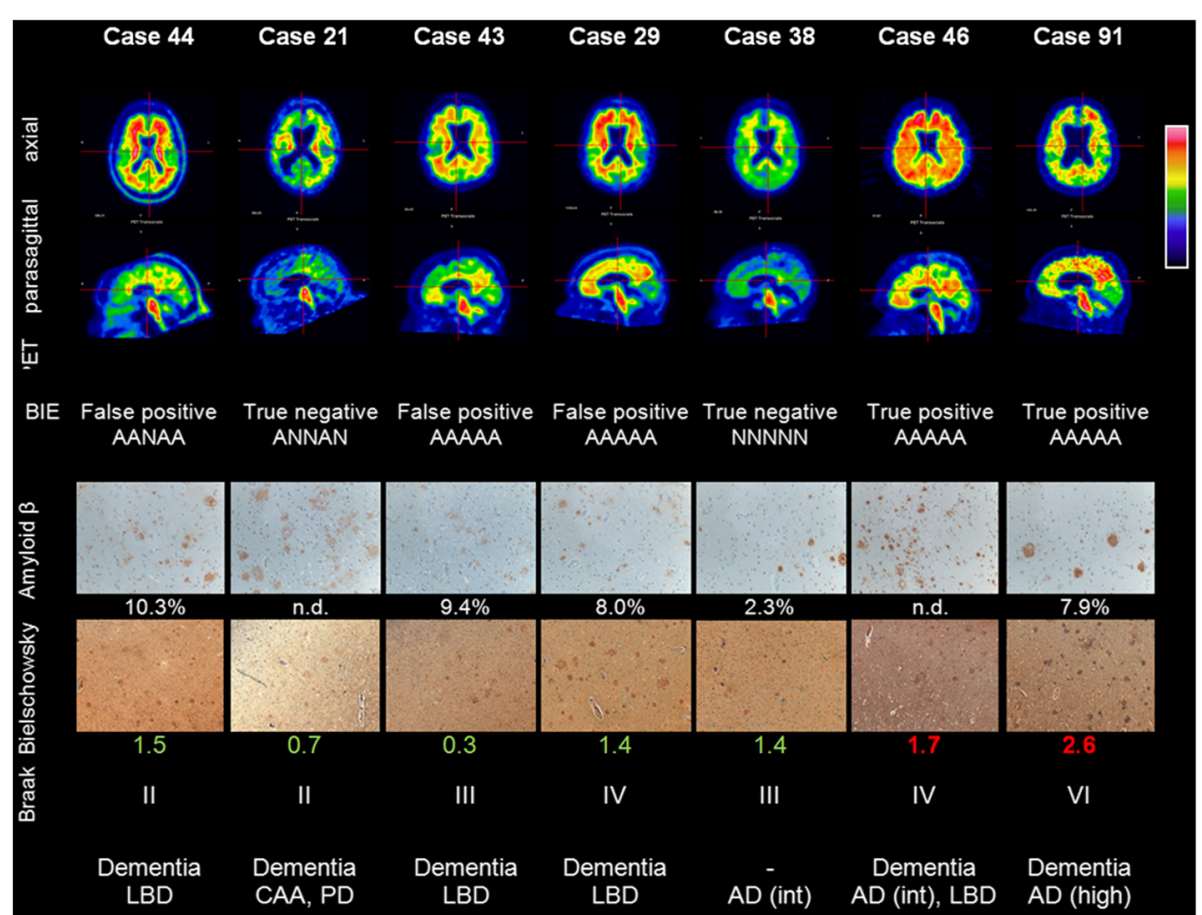

Fig. 3 PET images and representative histopathology for a range of subjects including some disparity cases (PET images are representative Rainbow colour scale, axial and parasagittal slices. BIE status and results for the 5 readers ( $N=$ normal, $A=a b n o r m a l)$. A representative photomicrograph of $\beta$ amyloid IHC (frontal lobe) with \% area (if determined), representative photomicrograph of Bielschowsky silver stain (frontal lobe) and Bielschowsky score (original magnification 100x for both); Braak stage of neurofibrillary tangles and overall neuropathological diagnosis, including AD likelihood (NIA-RI criteria). Note: the photomicrographs of the frontal lobe may not accurately represent the pathology of other regions

0.001 Kruskal-Wallis). Despite the temporal cortex being the most involved of the cortical regions by pathology, the temporal cortex was scored as abnormal by PET the least frequently of the cortical regions assessed. Inter-reader agreement and probability of a positive BIE assessment were lowest in the temporal cortex and in the striatum, although these differences did not reach statistical significance (Kruskal Wallis; $P>0.05)$. Observed regional BIE assessments were significantly different from those expected based upon
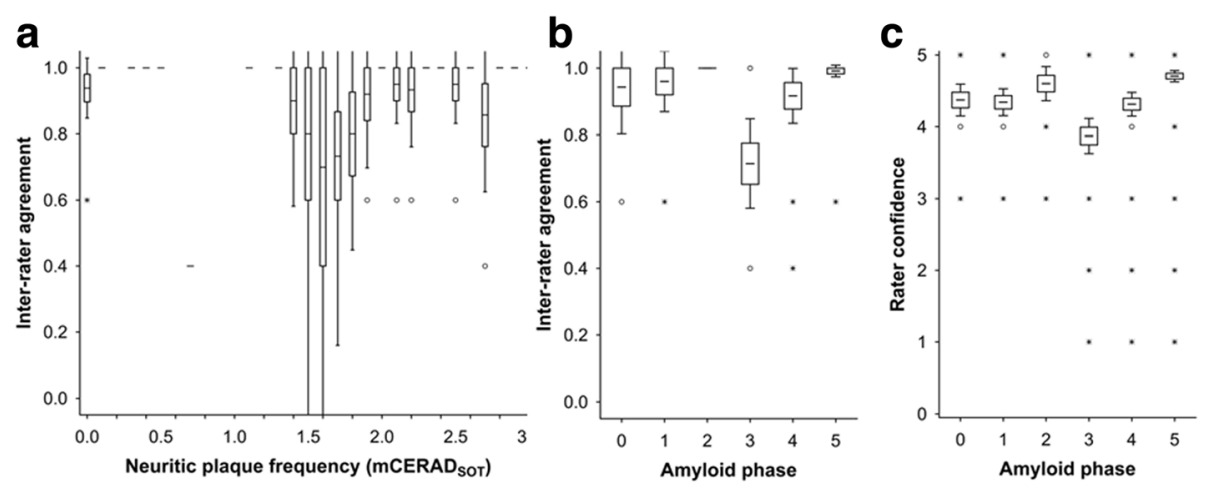

Fig. 4 Inter-reader agreement and reader confidence are decreased in cases with a plaque burden close to the threshold of PET detection. Panel

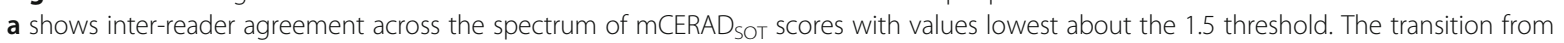
amyloid phase 2 to phase 3 is associated with neocortical plaque burden between sparse and moderate (see Fig. 4) and both inter-reader agreement (Panel b) and reader confidence (Panel $\mathbf{c})$ is lowest in phase $3(P<0.001$ for both data sets, Kruskal Wallis test). Inter-reader agreement was determined as Fleiss' kappa coefficient $P_{i}$ (see text for details) and for 5 readers is 1 when all 5 readers are in agreement, 0.6 when the agreement is 4:1 and 0.4 when the agreement is split 3:2. Confidence was recorded as a 5 point scale (1-5) with 5 being most certain and 1 being the least certain. For all panels, boxes represent mean $+/-1$ standard error and whiskers represent $95 \%$ confidence interval. Open circles represent outlier values and asterisks represent extreme values (see Materials and methods for details) 

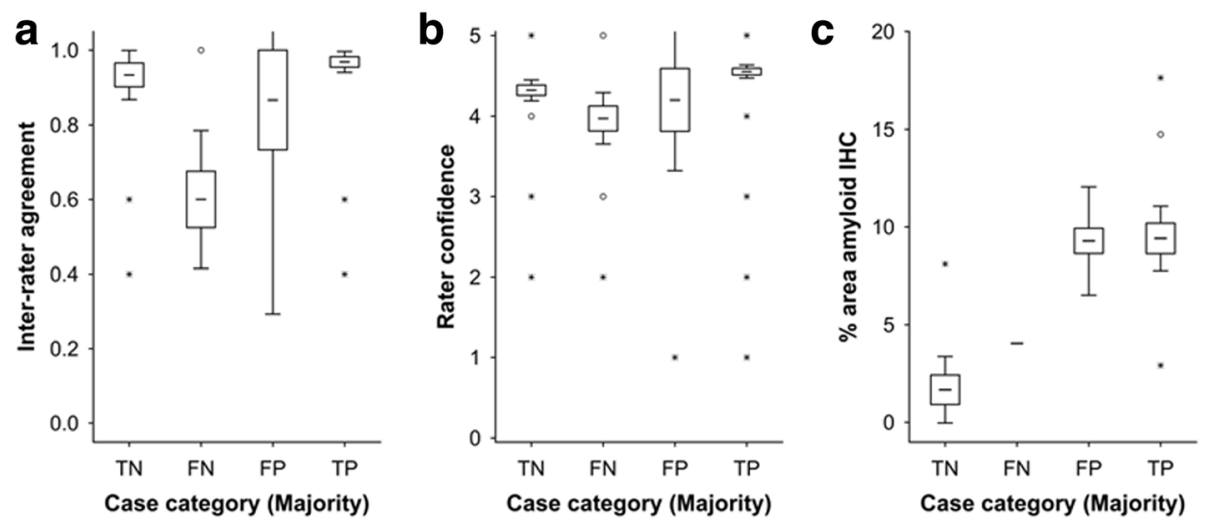

Fig. 5 Cases in which there was disparity between the pathology dichotomy as normal or abnormal and PET negative or positive were associated with low reader confidence (Panel a) and inter-reader agreement (Panel b). False-negative cases (FN) where the majority interpreted the PET image as negative in the presence of an abnormal neuritic plaque burden were associated with low inter-reader agreement ( $P<0.0001$, Kruskal Wallis test). False-positive (FP) cases were associated with low reader confidence ( $P<0.0001$, Kruskal Wallis test). Inter-reader agreement was determined as Fleiss' kappa coefficient $P_{i}$ (see text for details) which for 5 readers is 1 when all 5 readers are in agreement, 0.6 when the agreement is $4: 1$ and 0.4 when the agreement is split 3:2. Reader confidence was recorded as a 5-point scale (1-5) with 5 being most certain and 1 being the least certain. Panel $\mathbf{c}$ In the 3 false-positive cases, although neuritic plaque frequency was below threshold, $\beta$-amyloid in the form of diffuse plaques was comparable to mCERADsot cases (true positives; TP). One case also had high \% area stained for $\beta$-amyloid by IHC but was low intensity and the readers called this case true negative (TN) (reader ratio 5:0, Case 13) suggesting that in this case cortical $\beta$-amyloid levels in the absence of any neuritic plaques were insufficient to pro-

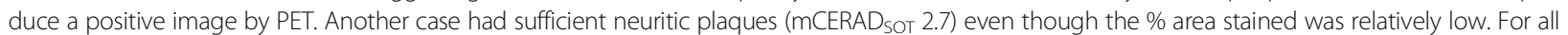
panels, boxes represent mean +/- 1 standard error and whiskers represent 95\% confidence interval. Open circles represent outlier values and asterisks represent extreme values (see Materials and methods for details)

the regional mCERAD SOT $_{\text {scores }}\left(P=0.0079 ; X^{2}\right.$ test $)$ with the temporal lobe assessed as abnormal less frequently than expected, and the other three cortical regions scored as abnormal slightly more frequently than expected. Together, these results possibly reflect a slightly more difficult interpretation of the temporal lobes compared to the other regions because of the combination of depth of sulci and partial volume effects.

Asymmetry was an occasional feature in the PET images, but while there was a small potential to misclassify cases if the $\beta$-amyloid burden was above threshold in the hemisphere not sampled for histopathology assessment, asymmetry was usually focal and no images showed asymmetry in all regions assessed (global asymmetry). Furthermore, there were very few cases dichotomized as abnormal by either PET or pathology based on a single region (which would be the cases most susceptible to misclassification), and so the risk of misclassification appeared small. Nevertheless, all cases where there was notable asymmetry (as determined post-hoc) were assessed for this potential. A potential to misclassify was only present under two circumstances. First, if pathology was normal in the left hemisphere but the PET image was assessed as abnormal, this could be incorrectly classified as a false positive if the pathologically unassessed right hemisphere was the basis for calling the image positive. However, there were no subjects with this combination. Second, if pathology (based on the left hemisphere) and PET assessments (based on both hemispheres) were both negative but there was $\beta$ amyloid pathology in the right hemisphere, then the case might have been wrongly categorized as a true negative when it would actually have been a false negative. Of five cases meeting this criterion, 4 showed minimal $\beta$-amyloid pathology; $\mathrm{mCERAD}_{\mathrm{SOT}}$ values were $0,0,0$ and 0.4 , and all readers recorded negative PET assessments for all regions. One subject (case 39) did have left-sided cortical $\beta$-amyloid close to the threshold, but again all readers uniformly recorded negative cortex assessments from the bilateral $\left[{ }^{18} \mathrm{~F}\right]$ flutemetamol image. The literature also suggests that there is general bilateral symmetry for $\beta$-amyloid by both PET [48] and pathology [44].

\section{Amyloid in neuritic plaques is the predominant $\beta$-amyloid pathology imaged by PET}

The assessment of neuritic plaques in neocortical regions of the brains has been a key feature for the diagnosis of AD and was expected to be the predominant histopathological feature correlating with $\left[{ }^{18} \mathrm{~F}\right]$ flutemetamol PET image assessment. CERAD, $\mathrm{mCERAD}_{\mathrm{SOT}}$, and $\mathrm{mCERAD}_{\text {mode }}$ neuritic plaque assessments generally agreed, although the single-point estimate CERAD included disparities with mCERAD $_{\text {SOT }}$ in $15 / 106$ cases $(14 \%)$ and was a less robust predictor of PET image positivity than both $\mathrm{mCERAD}_{\mathrm{SOT}}$ and mCERADmode (Table 5). Four cases (4\%) were dichotomised 
Table 5 Summary statistics and case status for each of the $\beta$-amyloid dichotomy algorithms

\begin{tabular}{|c|c|c|c|c|c|c|c|c|}
\hline \multirow[b]{2}{*}{ Reference standard } & \multicolumn{4}{|c|}{ Case status (5 readers per case) } & \multicolumn{4}{|c|}{ Pathology } \\
\hline & $\mathrm{TN}$ & FP & $\mathrm{FN}$ & TP & Normal & Abnormal & Sensitivity $^{a}$ & Specificity $^{b}$ \\
\hline mCERAD & 131 & 19 & 33 & 347 & 30 & 76 & $91 \%$ & $87 \%$ \\
\hline mCERAD ${ }_{\text {mode }}^{d}$ & 134 & 26 & 30 & 340 & 32 & 74 & $92 \%$ & $84 \%$ \\
\hline CERAD $^{\mathrm{e}}$ & 134 & 51 & 30 & 315 & 37 & 69 & $91 \%$ & $72 \%$ \\
\hline Amyloid phase $^{f}$ & 108 & 2 & 56 & 364 & 22 & 84 & $87 \%$ & $98 \%$ \\
\hline
\end{tabular}

$T N$ true negative, $F P$ false positive, $F N$ false negative, $T P$ true positive

${ }^{a}$ sensitivity (true positive rate) $=\mathrm{TP} /(\mathrm{TP}+\mathrm{FN})$

${ }^{\mathrm{b}}$ specificity (true negative rate) $=\mathrm{TN} /(\mathrm{TN}+\mathrm{FP})$

${ }^{c}$ Abnormal defined as any regional mCERAD $_{\text {SOT }}$ score $>1.5$ ). Note while these analyses are presented by individual reads, the $a$ priori analysis was by majority read (sensitivity was $91 \%$ and specificity was $90 \%$ by majority read $\mathrm{N}=106$ )

${ }^{\mathrm{d}}$ Abnormal defined as any CERAD region moderate or frequent (multiple measures)

${ }^{\mathrm{e}}$ Abnormal defined as and CERAD region moderate or frequent (single point estimate)

${ }^{f}$ Abnormal defined as phase 3,4 or 5

differently when using mCERAD $\mathrm{SOT}_{\mathrm{S}}$ vs. mCERAD mode. Significant plaque burdens in non-CERAD regions led to the differential classification of only two cases (cases 26 and 74), supporting the general view that $\beta$-amyloid pathology limited to non-CERAD regions (anterior cingulate and primary visual cortex in these cases) is atypical (2/ $106=2 \%$ ), but should not be ignored when comparing to global cortical assessments used in $\beta$-amyloid PET imaging. Two further cases were dichotomised differently due to the statistical method used (mean vs. mode) in subjects with borderline $\beta$-amyloid pathology (cases 32 and 44). Indeed, discrepancies were only noted between any of the three neuritic plaque assessment methods when the burden was close to the threshold between sparse and moderate. Since these differences were so few in number and changed from false classifications (false negative, FN; false positive, $\mathrm{FP}$ ) to true classifications (true negative, $\mathrm{TN}$; true positive, TP) (and vice versa; case $26 \mathrm{FP} \rightarrow \mathrm{TN}$, case $32 \mathrm{TN} \rightarrow \mathrm{FN}$, case $44 \mathrm{FP} \rightarrow \mathrm{TP}$, and case $74 \mathrm{FN} \rightarrow \mathrm{TN}$; mCERAD $_{\mathrm{SOT}} \rightarrow$ mCERAD $_{\text {mode }}$ respectively), sensitivity and specificity of majority PET reads were comparable for the 2 methods (respectively $91 \%$ and $90 \%$ for mCERADsot and $92 \%$ and $87.5 \%$ for mCERAD $_{\text {mode }}$ ). These performance measures were superior to those using the single-point estimate CERAD assessment (91\% and 76\% for sensitivity and specificity, respectively).

Comparison of CERAD single-point estimates and mCERAD ${ }_{\text {SOT }}$ showed 15 disparate global assessments, all of which were in the sparse or moderate categories and close to the threshold between normal and abnormal. This supports the superiority of repeated measures over a single (or even duplicate) measure, but also highlights the potential for misclassification in any biological continuum when close to a fixed threshold. The fact that our SOT methods and the CERAD single point estimates all correlate well with PET tracer retention dichotomy strongly supports the notion that neuritic plaque burden is strongly associated with PET $\beta$ amyloid imaging. The probability of a subject having a positive PET image interpretation increased with neuritic plaque burden (Fig. 2a). Subjects with a sparse neuritic plaque frequency were equally as likely to have a positive or negative PET image, suggesting that the actual threshold of plaque burden that can be detected by $\left[{ }^{18} \mathrm{~F}\right]$ flutemetamol (between sparse and moderate) is below the one generally accepted to be diagnostically relevant for $\mathrm{AD}$ diagnosis (moderate or frequent) [41]. Both cortical neuritic (Bielschowsky) and total ( $\beta$-amyloid IHC) plaque burden was higher in the neocortex with advancing disease progression as determined by the $\beta$-amyloid phase [58] (Fig. 6) when measured as a single CERAD categorical point estimate or by mCERAD SOT (Bielschowsky for neuritic plaques) or CERAD-like categorical score ( $\beta$-amyloid IHC for diffuse plaques). This increase in cortical plaque burden was associated with an increase in the probability of positive PET assessment starting in phase 3 with near certainty in phase 5. The implication is that although neocortical plaques are present in phases 1 and 2, these are below the limit of detection by blinded visual assessment of $\left[{ }^{18} \mathrm{~F}\right]$ flutemetamol PET images and that the threshold of a negative or positive PET image is reached between phase 3 and phase 4 . These data also illustrate the increasing cortical $\beta$-amyloid burden associated with increasing subcortical $\beta$-amyloid distribution.

The maximal regional mCERAD ${ }_{\mathrm{SOT}}$ score was observed most frequently in the middle temporal lobe in nearly half of all abnormal cases (Table 6). Other CERAD regions were also frequently observed to be the region of maximal involvement. While the precuneus was assessed as positive by PET in all BIE-abnormal cases, this region was only classified as abnormal by pathology in $75 \%$ of the cases globally classified as abnormal. Pathology in other regions was variable. $\beta$-amyloid burden in the primary visual cortex was highly variable. The maximal involvement of the CERAD regions is in broad agreement with accumulated histopathological studies that indicate a progressive spread of $\beta$-amyloid deposition within the brain, beginning in the 

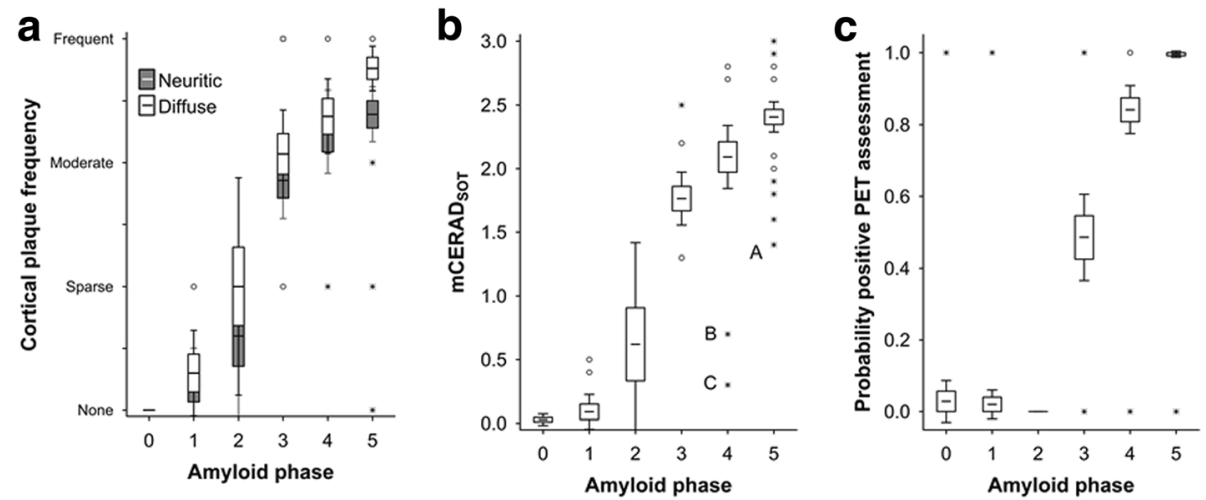

Fig. 6 Neocortical diffuse and neuritic plaque frequency and probability of PET positive interpretation increases with $\beta$-amyloid phase. Amyloid phase represents a progression of plaque deposition with advancing AD starting in the neocortex (phase 0-2) and progressing into the midbrain (phase 3) and hindbrain (phase 5). By phase 4, neocortical neuritic plaques are sufficiently abundant to be detectable by PET imaging. Panel $\mathbf{a}$. The abundance of both diffuse and neuritic plaques increase with advancing amyloid phase. The plots represent mean neocortical plaque frequencies $(0=$ none, $1=$ sparse, $2=$ moderate and $3=$ frequent) per amyloid phase and plaque frequency is determined by the CERAD single point estimate for each subject ( $N=106)$. Panel

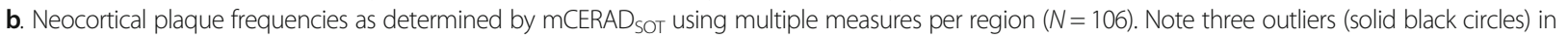
phase $4 / 5$ with mCERAD ${ }_{\text {SOT }}$ below 1.5 meaning that these cases were considered normal by mCERAD ${ }_{\text {SOT }}$ but abnormal by amyloid phase. Two of these cases (A and C) were false positives by majority, while B was true negative by majority, but only by a 3:2 reader split. Panel $\mathbf{c}$. The probability of positive global PET assessment increases with amyloid phase ( $N=5$ for each subject, $N=35,50,25,70,120$ and 230 for phases $0-5$ respectively). For all panels, boxes represent mean +/- 1 standard error and whiskers represent 95\% confidence interval. Open circles represent outlier values and asterisks represent extreme values (see Materials and methods for details)

lateral neocortex and basal neocortex [7] and spreading through medial neocortex, subcortical diencephalon, midbrain and then into the cerebellar and hindbrain structures [58]. These studies have also suggested that most neocortical regions have a heavy burden by the time the disease has progressed to symptomatic phases. While the frequencies of $\beta$-amyloid abnormality $\left(\right.$ mCERAD $\left._{\mathrm{SOT}}>1.5\right)$ were greatest in CERAD regions (Table 6), there was no significant difference in mean MCERAD $_{\mathrm{SOT}}$ between the regions $(P=0.68$; Kruskal-Wallis test $N=848)$. Of the 76 abnormal cases by mCERAD ${ }_{\mathrm{SOT}}, 40(53 \%)$ were abnormal in all eight cortical regions and 70 (92\%) were abnormal in multiple regions. Only 6 cases $(8 \%)$ were dichotomised as abnormal on the basis of a single region and were therefore theoretically more prone to misclassification. Indeed, $3 / 6$ of these single region dichotomies were discordant with PET (i.e. false-negative PET assessment by majority).

Analysis of the various histopathological scores against PET assessment showed superiority of plaque based assessments over neuropathological diagnoses. ROC analysis was performed for BIE dichotomy against categorical scores for CERAD (none, sparse, moderate, frequent), NIA-RI AD likelihood (not, low, intermediate high), NIA-AA AD neuropathologic changes (none, low, intermediate, high) and amyloid phase (phase 0-5) and for dichotomised neuropathology (moderate or frequent CERAD, intermediate or

Table 6 Frequency of regional $\beta$-amyloid abnormality

\begin{tabular}{|c|c|c|c|c|c|c|c|c|}
\hline & \multicolumn{8}{|c|}{ Neocortical region } \\
\hline & \multicolumn{4}{|c|}{ CERAD regions } & \multirow[b]{2}{*}{ ACG } & \multirow[b]{2}{*}{ PCG } & \multirow[b]{2}{*}{ PRC } & \multirow[b]{2}{*}{ PVC } \\
\hline & MFL & STG & MTG & $\mathrm{IPL}$ & & & & \\
\hline \multicolumn{9}{|l|}{ Pathology } \\
\hline Frequency region maximal $^{a}$ & $13(17 \%)$ & $5(7 \%)$ & $33(43 \%)$ & $19(25 \%)$ & $8(11 \%)$ & $6(8 \%)$ & $12(16 \%)$ & $17(22 \%)$ \\
\hline $\begin{array}{l}\text { Frequency region abnormal mCERAD } \\
\text { (of } 76 \text { abnormal cases) }\end{array}$ & $61(80 \%)$ & $57(75 \%)$ & $65(85 \%)$ & $66(87 \%)$ & $56(74 \%)$ & $63(83 \%)$ & $57(75 \%)$ & $60(79 \%)$ \\
\hline $\mathrm{PET}^{\mathrm{b}}$ & Frontal $^{c}$ & Temporal & & Parietal & & PCG/PRC & & \\
\hline $\begin{array}{l}\text { Frequency abnormal } 5 \text { reads per case } \\
\text { (of } 71 \text { abnormal cases by majority) }\end{array}$ & $326(92 \%)$ & $299(84 \%)$ & & $323(91 \%)$ & & $331(93 \%)$ & & \\
\hline
\end{tabular}

MFL midfrontal lobe, STG superior temporal gyrus, MTG middle temporal gyrus, IPL inferior parietal lobe, ACG anterior cingulate gyrus, PCG posterior cingulate gyrus, $P R C$ Precuneus, PVC primary visual cortex

${ }^{a}$ multiple regions may be simultaneously the region of maximal involvement

${ }^{b}$ BIE assessment also included the subcortical striatal region which was abnormal in 302 assessments (85\%)

'The frontal lobe was also assessed with the anterior-most aspect of the anterior cingulate in BIE assessment 
high NIA-RI and NIA-AA, and phase 3 or more Thal amyloid phase) against composite cortical SUVRs. In both sets of analyses Thal amyloid phase gave the greatest areas under the curve $(>0.95)$ and this was slightly superior to the ROC analysis of dichotomised BIE against the continuous variable mCERAD $_{\mathrm{SOT}}$ of 0.949 . (see Additional files 2, 3, 4, 5 and 6).

\section{The lower limit of $\beta$-amyloid detection by PET is of diagnostic relevance}

Three observations indicate that the lower limit of detection of plaques by PET is just below the threshold of plaque burden used for the diagnosis of $\mathrm{AD}$, i.e. the lower limit for the detection of plaques by $\left[{ }^{18} \mathrm{~F}\right]$ flutemetamol appears to be between the CERAD categories of sparse and moderate (5-6 neuritic plaques per 100x FoV). Firstly, the probability of an abnormal scan interpretation is almost 0 for the CERAD category of none, and almost 1 for the categories of moderate and frequent, while it is approximately 0.5 for the sparse category (Fig. 2a). Secondly, analysis of regional mCERADsOT values shows that the probability of a region being assessed as abnormal by PET increases between sparse and the mid-point between sparse $\left(\mathrm{mCERAD}_{\mathrm{SoT}}=1.0\right)$ and moderate $\left(\mathrm{mCERAD}_{\mathrm{SoT}}=2.0\right)$, i.e. at $\mathrm{mCERAD}_{\mathrm{SoT}}=$ 1.5. Above a score of 1.5 , the probability plateaus (Fig. 2b). Thirdly, ROC analysis of mapped regional histometric endpoints (neuritic plaque density, $\beta$-amyloid IHC area) and SUVR measures in all 8 neocortical regions for a subset of 32 cases provided optimal sensitivity and specificity in the mCERAD $\mathrm{SOT}_{\mathrm{SO}}$ range of $0.9-1.1$, corresponding to approximately 2-3 neuritic plaques per $100 \mathrm{x} \mathrm{FoV}$, in the middle of the sparse CERAD category (Additional file 2) and representing $\beta$-amyloid levels that are on average lower than those associated with clinicopathologically significant $\mathrm{AD}$ (cognitive impairment or dementia due to $\mathrm{AD}$ ).

\section{$\beta$-amyloid is the primary amyloid target of $\left[{ }^{18} \mathrm{~F}\right]$ flutemetamol}

Although amyloid in neuritic plaque burden probably accounts for the majority of tracer retention, amyloid ( $\beta$ pleated sheet) structure is found in several protein aggregates within the brain in $\mathrm{AD}$ and other neuropathological degenerative processes. The binding mechanism of Thioflavin-T derivatives (such as flutemetamol) to $\beta$ sheets in $\beta$-amyloid fibrils is not specific to the primary peptide sequence $[6,41]$. These alternative amyloid deposits include other insoluble forms of $\beta$-amyloid (diffuse plaques and CAA), as well as other forms of amyloid, such as tau (neurofibrillary tangles [NFT]) and $\alpha$-synuclein (Lewy bodies). Our subject group contained 2 cases of tangle-predominant dementia and 1 case of Pick's disease, with minimal co-incidental $\beta$-amyloid involvement. All 3 cases were PET negative, suggesting that any cross reactivity to tau at these levels is insufficient to cause ambiguity to interpretation of $\beta$-amyloidbased tracer retention. Despite $\beta$-amyloid deposits being a commonly observed co-pathology in LBD subjects, only $55 \%$ (16/29 cases) were diagnosed with co-incident AD against the NIA-RI criteria, despite 79\% (23/29) being PET positive. Most LBD cases without $\beta$-amyloid pathology were PET negative and therefore $\alpha$-synuclein deposits per se may not have a strong contribution to $\beta$ amyloid PET imaging, as suggested by previous studies $[9,19,70]$. Of the 13 LBD cases that were not coincident with $\mathrm{AD}, 7$ were mCERAD $\mathrm{SOT}_{\text {SOT }}$ normal (4 PET negative and 3 PET positive) and 6 were mCERAD $\mathrm{SOT}_{\mathrm{SOT}}$ abnormal (5/6 PET positive). The 3 PET positive mCERAD $_{\text {SOT }}$ normal cases in this disease category represent the only false positive cases in the GE067-026 trial and were scrutinised in greater detail. Two of the three cases (cases 29 and 44) were close to the $>1.5$ a priori threshold for mCERAD ${ }_{\mathrm{SOT}}$. As the limit of detection is likely below this threshold (see above), are probably only false positive by virtue of this a priori threshold. However, the third case (case 43) had very few neuritic plaques and these were confined to the primary visual cortex, a region not directly assessed in the PET BIE. Two of these 3 cases had not had $\beta$-amyloid IHC \% area measurements, and so were added to the original 30 cases in a post hoc analysis. Both were determined to have a high percentage of tissue area occupied by $\beta$-amyloid (Table 1 ).

Only 3 cases of CAA were recorded in the absence of a diagnosis of $\mathrm{AD}$, and 2 of these were determined to be mCERAD $_{\text {SOT }}$ normal. Both were PET negative. For one case CAA involvement was focal and minimal, whereas the other was more extensive, i.e. Vonsattel grade 2, stage 2 , and type $1[56,64]$. With so few cases of CAA in the absence of $\mathrm{AD}$ (or accompanying plaque burden), no firm inference can be made regarding the contribution of CAA to tracer retention. Furthermore, composite $S U_{\text {cer }}$ of $A D$ subjects with and without CAA were comparable $(P>0.5$, ANOVA). However, indirect evidence that CAA might add to cortical tracer retention may be seen in cases where cortical $\beta$-amyloid in the form of neuritic plaques is already close to but below the threshold of detection, i.e. in the 14 amyloid phase 3 cases. In this small subset, CAA was recorded in all 8 cases in which the majority BIE assessment was abnormal and was absent in 5/6 cases where the BIE assessment was normal. The remaining case with majority normal BIE had only focal CAA. The mCERAD SOT scores for these 14 cases were comparable, while the probability of a BIE assessment as abnormal and the cortical composite SUVRs were increased (Fig. 7).

Although the contribution of diffuse plaques to tracer retention is difficult to determine precisely, 2 


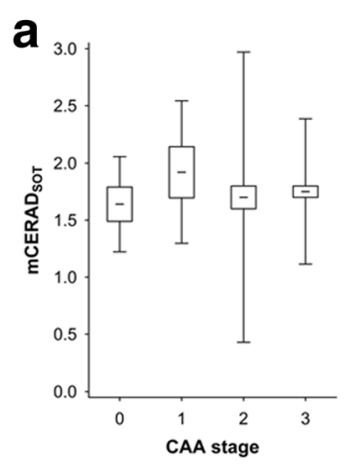

d

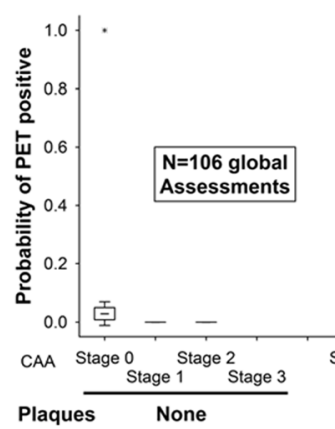

e

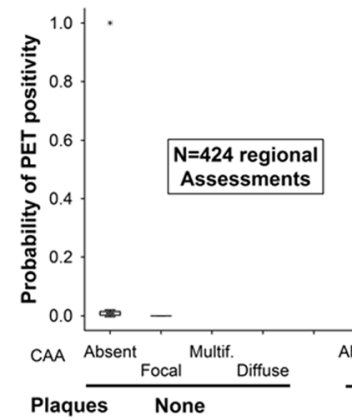

b

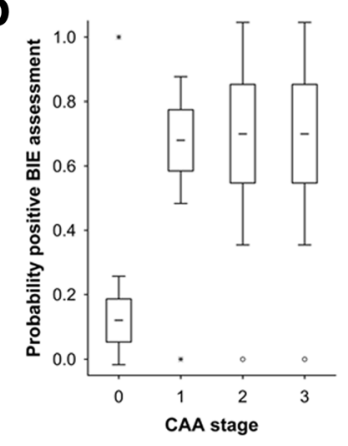

.
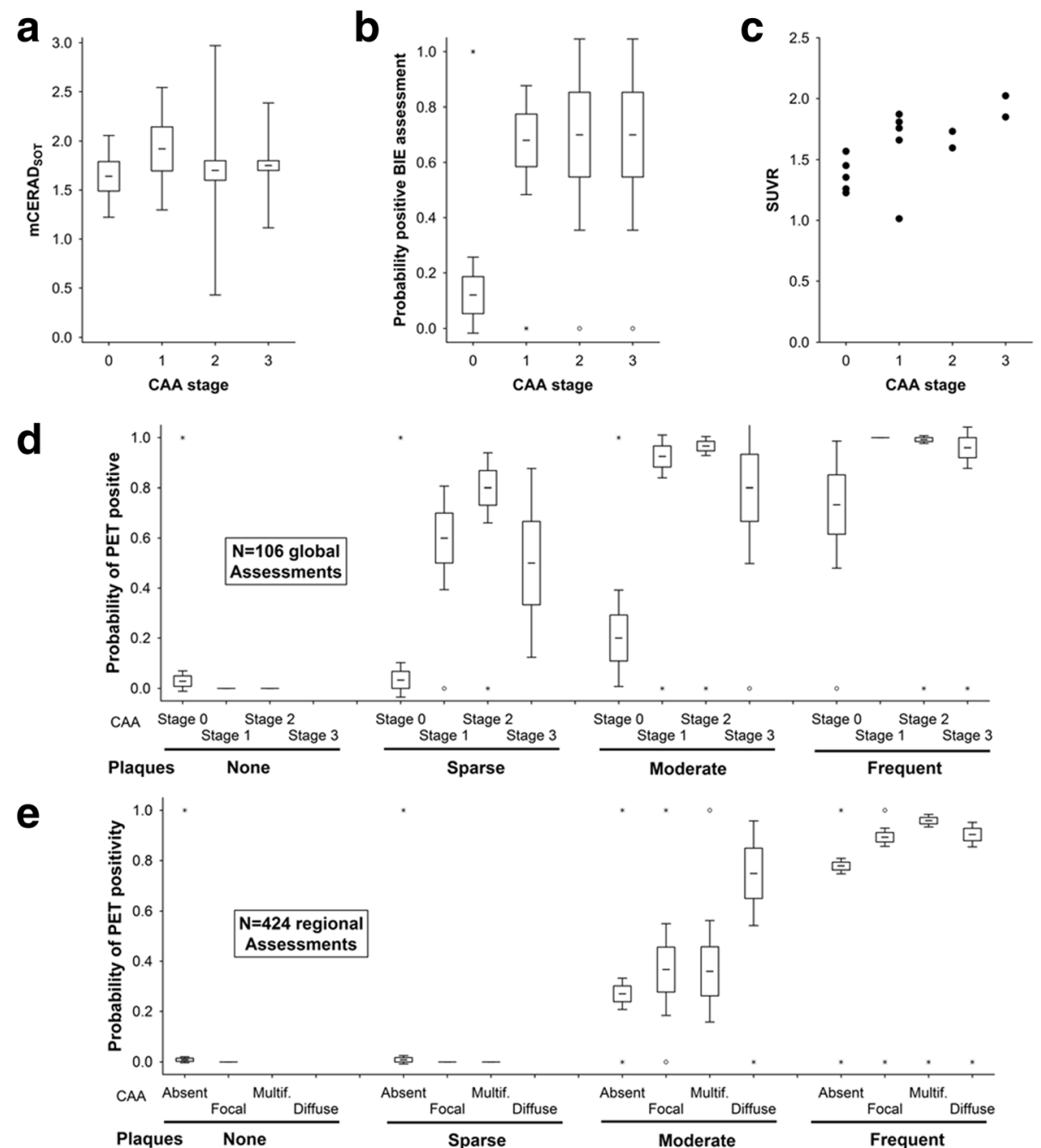

Fig. 7 CAA contributes weakly to PET cortical positivity. In amyloid phase 3 cases, where cortical neuritic plaque load is borderline (Panel a), PET assessment as abnormal is more likely $(P=0.0001$, Kuskal Wallis test) in the presence of CAA (Panel $\mathbf{b})$ and SUVR is elevated (Panel $\mathbf{c}, P<0.05$ Spearman). Panel d. Probability of positive BIE assessment for all subjects demonstrating that this analysis is only possible in cases with a modest plaque burden (Moderate or phase 3 cases) because of a lack of amyloid angiopathy in phase $0-2$ and certain cortical positivity due to abundant cortical neuritic plaques in Phase 4 and 5. Panel e. A similar pattern if observed with regional analysis (frontal, temporal, parietal and striatum). It is likely that this effect is subtle and additive to a neocortical plaque burden already close to the threshold. Boxes represent mean $+/-1$ standard error and whiskers represent 95\% confidence interval. Open circles represent outlier values and asterisks represent extreme values (see Materials and methods for details)

observations suggest that there is significant binding to diffuse plaques in this study. Firstly, the unequivocally positive PET assessment of case 43 in the virtual absence of neuritic plaques or CAA is possibly explained by the significant cortical diffuse plaque burden, $9.4 \%$ of the grey matter stained with $\beta$-amyloid IHC (Fig. 6, Panel c). Furthermore, any plaque burden in the striatum is predominantly in the form of diffuse plaques [20] and this region was frequently determined to be PET abnormal in our cohort. The term diffuse plaque covers a range of lesions from fleecy amorphous $\beta$-amyloid deposits through to plaques that are morphologically better defined but lack the dense core or dystrophic neurites required to be identified as neuritic. Although it has become commonly assumed that diffuse plaques do not contain fibrillar amyloid, electron microscopic observations have indicated that diffuse plaques contain sparse, loosely-textured amyloid fibrils [69] and many diffuse plaques fluoresce weakly with thioflavin S [15] indicating that fibrillar amyloid is often present, although at lower densities than within neuritic or core-only plaques. Also, while the concentration of fibrillar $\beta$-amyloid may be substantially lower within diffuse, as opposed to neuritic plaques, the volume occupied by diffuse plaques can be many times greater [43]. These observations support that, while being uncommon occurrence, a 
heavy diffuse plaque burden in the absence of neuritic plaques may be sufficient to generate an abnormal tracer retention signal.

\section{Where pathology and PET imaging disagree}

In our cohort of 106 subjects, the majority BIE read was disparate from the neuritic plaque assessment in 10 cases, 3 of which were false positives (abnormal PET image with normal pathology) and 7 of which were false negatives (normal PET images with abnormal pathology). Of these 10 cases, half had neuritic plaque density scores near the threshold (see below), but in the other 5 cases (cases 27, 28, 41, 42 and 43) there was no overt reason for the disparity, as the neuritic plaque density score was not considered borderline. Only one of the unexplained cases (case 43) was a false positive without a significant neuritic plaque burden (mCERADSOT $=0.3$, see earlier comments on heavy diffuse plaque burden in this case). A CERAD assessment of moderate entered onto the case report form was not substantiated by the mCERAD $D_{\mathrm{SOT}}$ score nor the neuropathology report, and was deemed a transcription error from "moderate diffuse plaque frequency," which was recorded in the report. However, whilst there is little doubt that the cortical plaque burden was low, the \% greymatter area stained with $\beta$-amyloid IHC was high (see Fig. 3), the amyloid phase was 4, the Braak stage was III, and the patient had a history of dementia. Because of the scarcity of neuritic plaques and presence of significant Lewy bodies, the primary diagnosis for this case was LBD. Interestingly, this case would be considered as having intermediate $\mathrm{AD}$ neuropathology changes if using the more recent National Institute on Aging-Alzheimer's Association (NIA-AA) diagnostic criteria [25].

The remaining 4 cases were false negatives with unequivocally abnormal $\beta$-amyloid burdens. The mCER$\mathrm{AD}_{\mathrm{SO}}$ scores for these cases were 1.8, 2.1, 1.9, and 2.7 for cases $27,28,41$, and 42 , respectively (Table 1 ). It is possible that the increased partial volume effect produced by severe neocortical atrophy may be at least partially responsible for the misclassification, although equivalent atrophy was seen in many patients whose images were correctly interpreted. Three of these 4 cases had a modest neuritic plaque burden and only one of them had a heavy neuritic plaque burden $\left(\mathrm{mCERAD}_{\mathrm{SOT}}=2.7\right.$, case 42). This last case should not have escaped PET detection. Indeed, in this case (case 42), 2/5 readers recorded abnormal retention in 4 of the 5 regions assessed, and all 5 readers reported low confidence in interpreting this image.

Most disparities occurred in cases with sparse or moderate neuritic plaque densities. The differentiation between sparse (1 to 5 plaques) and moderate (6 to 19 plaques) may differ by one plaque per field of view, and some misclassification is therefore inevitable when the underlying pathology is close to a threshold. Indeed, half of the disparity cases had $\beta$-amyloid pathology categories straddling the threshold for dichotomy (sparse and moderate) and amyloid phase $3(4 / 10)$. Of the 7 false negative cases, only $1 / 7$ (14\%) was abnormal for all 8 cortical regions assessed compared to $39 / 69$ (56\%) in true positive cases. The remaining cases varied from 1 to 5 regions abnormal (out of the 8 sampled). Furthermore, of 6 cases classified as abnormal on the basis of abnormal pathology in a single region, half were classified as false negatives. However, it is important to determine whether additional factors could contribute to misclassification.

In a post-hoc analysis, we classified cases as equivocal by either PET or pathology against the following defined criteria; equivocal by pathology if the $\mathrm{mCERAD}_{\mathrm{SOT}} \max$ was $1.25-1.75$, based on a theoretical influence of 1 aberrant measure on a regional assessment; 5 slides scored as 1.5 and one slide scored as $0\left(\mathrm{mCERAD}_{\text {SOT }}=\right.$ 1.25 ) or 3 (mCERAD SOT $_{1.75)}$; equivocal by PET if fewer than $4 / 5$ readers concurred; i.e., if majority assessment was 3:2, or if reader confidence was low (mean confidence of less than 4 on a scale of 1-5). A total of 24 cases were equivocal by either PET or pathology criteria; 6 cases were equivocal by both criteria. Fourteen cases were considered as equivocal by pathology, 12 with borderline mCERAD ${ }_{\mathrm{SOT}}$ and 6 with mCERAD $_{\mathrm{SOT}}>1.5$ based on a single region (4 cases met both criteria). Sixteen cases were equivocal by PET, all with low reader confidence and 4 of these also by split majority (3:2 reader ratio). Interestingly, of these 16 cases, half were amyloid phase 3 , or the preclinical to clinical transition phase $\left(P<0.01, X^{2}\right.$ test; observed vs. expected), indicating that at this intermediate level of $\mathrm{AD} \beta$-amyloid pathology, the images are less easy to interpret. Indeed, of the 14 phase 3 cases, only half were interpreted as positive by BIE majority. Of the 10 cases that were false positive or false negative by PET majority read, half were equivocal by pathology.

\section{Discussion}

The data presented in this study examined the histopathology underlying $\left[{ }^{18} \mathrm{~F}\right]$ flutemetamol tracer retention and PET image interpretation. The cohort of 106 endof-life subjects represented a broad and continuous spectrum of $\beta$-amyloid pathology and other comorbidities, including several cases where the neuritic plaque burden was diagnostically equivocal. Such cases are valuable for a critical assessment of imaging agent performance and diagnostic limitations.

No subjects were designated as abnormal by majority PET interpretation in the absence of fibrillar $\beta$-amyloid plaques. This study, and others using alternative $\beta$ amyloid PET tracers, demonstrate that neocortical 
neuritic plaque burden is the most important $\beta$-amyloid histopathological feature underlying tracer retention [11]. Key to demonstrating this was the reliable assessment of the neuritic plaque burden through multiple regional assessments that considerably improved upon a single point CERAD assessment, which is vulnerable to sampling bias and inter-reader variability [1]. Additionally, rather than limiting histopathological or PET analysis to the 3 anatomic regions originally recommended by CERAD, global cerebral tracer retention, which is reflective of global cerebral $\beta$-amyloid deposition, is validated by the good efficacy of multiple trials and tracers $[11,12,28$, 31, 35-38, 47, 50-52, 65-67]. Indeed, interpretation of PET images may be enhanced by the inclusion of medial cerebral gyri, notably the cingulate and precuneus, regions that have not previously formed part of the standard neuropathology assessment. In practice, assessment as abnormal based upon a single region is unusual. Only a single case $(<1 \%)$ in our cohort was designated as abnormal based upon a single positive PET region. Interestingly this case has borderline amyloid burden (case 49). Although tissue sections are relatively thin, in coronal cross section they cover a cortical area $(3-5 \mathrm{~cm})$ comparable to that assessed by PET and are unlikely to be a cause of discrepancy between the pathology and PET, especially if 8 (or more) cortical regions are assessed and multiple plaque measures per section also mitigated plaque heterogeneity. Furthermore, there is likely to be merit in assessment of subcortical areas such as the striatum [5] which is significant in amyloid progression [25, 57].

Unsurprisingly, the single most confounding factor in image interpretation is equivocal pathology, where the underlying histopathology is near the limit of reliable detection in PET images. In the present multi-reader study, this was manifest in low reader confidence and disagreement between the multiple readers. In clinical practice, it will probably be beneficial to consider a categorical assessment of "equivocal", an option which was not made available to readers during efficacy trials due to the regulatory requirement of dichotomy. Importantly, the detectable neuritic plaque burden by $\left[{ }^{18} \mathrm{~F}\right]$ flutemetamol imaging is diagnostically relevant in that it aligns with what is the accepted distinction between normal and pathologic levels of $\beta$-amyloid burden used for AD diagnosis. However, the probability of a positive PET assessment is non-zero in the sparse CERAD category and over a range of $\mathrm{mCERAD}_{\mathrm{SOT}}$ scores between 1 and 2 (sparse to moderate). It is possible that varying PET methodology may be able to alter the threshold for calling a scan positive. Paradoxically, better PET sensitivity will cause worse accuracy for predicting cognitive impairment due to $\mathrm{AD}$ as too many sparse-plaque cases will be called PET positive. On the other hand, detecting more of the sparse plaque cases may be better for subject selection for prevention trials for $\mathrm{AD}$, as the chance of success for such trials will presumably be better for those subjects at an earlier disease stage. The lower limit of detection of plaques by amyloid tracers may vary by anatomical region and partial volume effects, but it appears likely from these results that flutemetamol may detect cortical plaques below the threshold normally associated with a clinically significant "moderate" plaque burden and further quantitative methodological studies are needed.

Mixed pathologies in this cohort enabled us to investigate how co-incident pathology may impact PET image interpretation. Amyloidopathies other than those containing $\beta$-amyloid (i.e. tauopathies and synucleinopathies) did not clearly interfere with $\beta$-amyloid PET interpretation, most likely through a lack of detectable signal. We cannot comment on the potential for a weak signal in areas not assessed in this study, e.g. mesial temporal for Tau). However, other forms of $\beta$-amyloid deposits such as diffuse plaques and CAA possibly contribute to overall tracer retention. These forms of $\beta$-amyloid are often associated with $\mathrm{AD}$, but are also seen as separate entities, in that they are occasionally recorded in the absence of typical $A D$ neuritic plaque and NFT neuropathology. The $\beta$-amyloid burden in the form of diffuse plaques is variable, as the term covers a range of lesions with varying $\beta$-amyloid density and fibrillar $\beta$-amyloid content. Nonetheless, frequent diffuse plaques alone were sufficient for PET positivity in the cortex of one case (case 43) and in the striatum in 9 cases - in agreement with in vitro studies of flutemetamol and the chemically related $\mathrm{PiB}$, which lightly stain diffuse plaques [28].

While at first impression, the inability to discriminate between a significant diffuse plaque burden and a more modest neuritic plaque burden may appear to be a disadvantage, this may not be a critical weakness, as cases with a heavy load of cortical diffuse plaques but absent or scarce neuritic plaques are uncommon. Also, more importantly, such cases may meet current diagnostic criteria for clinicopathologically significant $A D$ as exemplified by case 43 , which met the 2012 AD criteria [25]. Indeed, such tracer retention by diffuse plaques may be a diagnostic advantage in regions such as the striatum, where plaques are predominantly diffuse [16] yet are diagnostically indicative of an advanced $\beta$ amyloid phase [58], and may also be used to predict associated NFT stage [16]. In this latter study, striatal $\beta$ amyloid identified AD pathology in the presence of Lewy body disease, which is analogous to some of our subjects. Striatal $\beta$-amyloid detection may be particularly important in some cases of presenilin-1 (PS1)-associated $\mathrm{AD}$ where the striatum may be the first area affected [34]. However, to our knowledge, we did not have any PS1 mutations in this study. The presence of plaques in 
the striatum represents a transition from amyloid phase 2 (Normal) to phase 3 (Abnormal) [58]. However, in the early stages of phase 3 amyloid burden is likely to be insufficient to be detected by PET imaging and the burden in the striatum may not become sufficient until phase 4 . Indeed, in the 6 cases where PET assessment was positive in the cortex but negative in the striatum 4 were classified as phase 3 and 2 were classified as phase 4 . We are currently assessing the significance of striatal imaging in further depth (see also changes to the $\mathrm{AD}$ diagnostic criteria below).

While the $\beta$-amyloid in cortical diffuse plaques may not often be clinically significant on its own, the deposits of $\beta$ amyloid in CAA confer a substantial risk of lobar haemorrhage. Therefore, the ability of $\beta$-amyloid tracers to identify CAA is of considerable interest [3,30]. While others have reported positive PET signal with CAA using the chemically related $\left[{ }^{11} \mathrm{C}\right] \mathrm{PiB}[3,30]$, our cohort contained no cases with CAA in the absence of at least some fibrillar $\beta$-amyloid plaques. Nonetheless, CAA may contribute to cerebral PET positivity in a subset of cases supporting reports that CAA is detectable by others [2] but that, although substantial amounts of $\beta$-amyloid may be present in vasculature, tracer retention by CAA is modest compared to that by cortical neuritic plaques. Our analysis did not include PET image assessment of the occipital lobe, where others report predominant CAA tracer retention [2]. Any potential clinical utility of $\beta$-amyloid tracers with regards to CAA may rest mainly in the ability to rule out CAA with a negative scan, in much the same way as a negative scan also rules out $\mathrm{AD}$.

Significant cortical atrophy in end-stage Alzheimer's disease can complicate the interpretation of $\left[{ }^{18} \mathrm{~F}\right]$ flutemetamol PET images, which requires assessment of pathological gray-matter retention adjacent to normal white-matter retention pattern. In most circumstances, this is relatively straightforward, but may be complicated by significant atrophy of the cortical ribbon, rendering differentiation between grey and white matter more difficult in the PET only image. As atrophy is a common feature of advanced AD, atrophy in the cohort as a whole was generally more frequent with increased positive PET images, reflecting the underlying advanced $\mathrm{AD}$. However, in cases where atrophy was recorded as severe, the probability of a positive PET interpretation decreased without a concurrent drop in reader confidence. Four of the seven false negative cases were noted to have severe atrophy during gross examination at post-mortem. It therefore appears likely that while equivocal pathology is associated with low reader confidence and likely accounts for some of the false negative cases - atrophy probably accounts for some of the false-negative interpretations without a concurrent drop in reader confidence. While amyloid pathology is generally symmetric in distribution, focal atrophy present in some of our cases showed distinct laterality, resulting in an asymmetric PET image. The electronic training program for image interpretation $[54,63]$ provides guidance on how to interpret images with such focal atrophy.

Our results demonstrate an association of $\left[{ }^{18} \mathrm{~F}\right]$ flutemetamol PET with $\beta$-amyloid deposition in the neocortex, while PET positivity correlated less well with a neuropathologically-supported diagnosis of $\mathrm{AD}$. The $\mathrm{AD}$ diagnostic criteria used here were the National Institute of Aging - Reagan Institute criteria [26]. The association between neuritic $\beta$-amyloid plaques and NFT is imperfect [21], and so any measure of $\beta$-amyloid in the cortex cannot 'rule in' AD, as it is unable to measure NFT. Instead, its utility is primarily intended to 'rule out' AD if the PET scan is negative. However, the PET detection of striatal plaque burden may offer a more sensitive assessment of AD progression [16] and a recent revision of AD diagnostic guidelines [25], which includes $\beta$-amyloid phase analysis [58], indicates that striatal imaging may refine $\mathrm{AD}$ assessment with $\beta$-amyloid tracers.

The GE067-026 trial findings presented here for $\left[{ }^{18} \mathrm{~F}\right]$ flutemetamol are consistent with those reported previously in cerebral biopsy trials and by other $\beta$-amyloid imaging agents in end-of-life subjects, such as the chemically related $\left[{ }^{11} \mathrm{C}\right] \mathrm{PiB}$ and chemically less similar $\left[{ }^{18} \mathrm{~F}\right] \mathrm{AV}-45$, supporting the utility of $\beta$-amyloid imaging in vivo [11, 28, 67]. The 'holy grail' for subject selection for prevention trials would be detection of early or preclinical plaque burden with a detection limit clearly below the levels that are associated with significant cognitive decline. The probability of a positive PET image interpretation in this study was $50 \%$ in the sparse category, suggesting that this may be possible, but it seems likely that analysis of other regions, such as the striatum or, better yet, a measurable, reliable quantification method, would likely improve the lower detection limit, enabling assessment of $\beta$ amyloid in preclinical AD, a stage of disease almost certainly more amenable to preventative treatment strategies or disease-modifying therapeutics. In this context, it is necessary to note that recent studies reported that amyloid PET only detects cases with already advanced amyloid phases (3 and higher) whereas initial phases (1 and 2) could not be identified with $\left[{ }^{18} \mathrm{~F}\right]$ flutemetamol or $\left[{ }^{11} \mathrm{C}\right] \mathrm{PIB}$ $[45,55]$. Conversely, increasing the sensitivity of PET $\beta$ amyloid scans may decrease the specificity to predict clinically significant AD neuropathology. It is possible that PET image interpretation methods might be adjustable to fit the clinical setting, with a less sensitive scan (similar to those currently in use) being used in the setting of dementia and a more sensitive scan used in the setting of selection of cognitively normal, $\beta$-amyloid positive subjects for $\mathrm{AD}$ prevention trials.

$\beta$-amyloid PET imaging studies with neuropathology validation in post-mortem brains $[2,9-11,27,28,32,33,45$, 
$53,62]$ or biopsy brain tissues $[37,50,51,67]$ are usually limited by small numbers of subjects and could be biased by variable time between PET acquisition and autopsy or biopsy. However, our study of 106 subjects is a relatively large cohort and the influence of variable PET to autopsy interval was not a significant factor [49]. Small numbers of disease transition subsets, such as 14 amyloid phase 3 subjects, show interesting results - low reader confidence, low inter-reader agreement and possible influence of CAA and while analyses reach statistical significance, conclusions drawn from small numbers such as these require further research to substantiate or refute these findings.

\section{Conclusions}

In summary, examination of data from an end-of-life clinical trial of Flutemetamol F 18 Injection demonstrated a high specificity and sensitivity for $\beta$-amyloid pathology, and detection of a diagnostically relevant neuritic plaque burden. Abnormal PET scans were always associated with underlying $\mathrm{AD}$ histopathology and equivocal PET scan reads usually indicated some underlying AD histopathology, possibly at levels usually associated with cognitive normality or early mild cognitive impairment. Other co-morbidities did not interfere with image interpretation, although advanced cortical atrophy could hamper interpretation even in advanced $\mathrm{AD}$ cases. For the future, opportunities to quantify or automate the interpretation of PET images [59] and the study of greater numbers of subjects in the intended target population may further refine the clinical utility of $\beta$-amyloid PET imaging following Flutemetamol F 18 Injection.

\section{Additional files}

Additional file 1: Example of the recording of pathology sampling to enable mapped cortical tracer retention SUVRs to be measured for correlation. Regions sampled from the lateral surface include the Midfrontal lobe (MFL), Superior Temporal Gyrus (STG), Middle Temporal Gyrus (MTG) and Inferior Parietal Lobe. Regions sampled from the medial surface include Anterior Cingulate Gyrus (ACG), Posterior Cingulate Gyrus (PCG), Precuneus (PRC) and Primary Visual Cortex (PVC). (DOC 90 kb)

Additional file 2: Receiver operator characteristic (ROC) analysis of regional histometric measures and standard retention value ratios. Regional analysis of 32 brains provided retention correlation with plaque burden and provided a large data set $(N=256)$ with which to estimate the lower limit of detection. For regional analysis SUVR baselines which varied between regions due to variable adjacent white-matter signal bleed were used to define a threshold for each region. Thresholds were determined post-hoc by ROC analysis as the maximal sum of the sensitivity (true positive rate) and specificity (1 - false-positive rate). (DOC $59 \mathrm{~kb}$ )

Additional file 3: ROC Analysis - Dichotomised BIE (majority read) vs categorical neuropathology. Comparison of the 4 histopathological criteria of assigning abnormal amyloid against a dichotomised (Abnormal/Normal) majority blinded PET Image evaluation. The 4 criteria shown are CERAD [43], National Institutes of Ageing - Reagan Institute AD diagnosis [26], National Institutes of Ageing - Alzheimer's Association AD diagnosis [25] and Amyloid phase (Phase 3 or greater) [58]. (DOC $201 \mathrm{~kb}$ )

Additional file 4: ROC Analysis: Dichotomised pathology vs continuous SUVR. Comparison of 5 histopathological criteria of assigning abnormal amyloid against PET signal quantification (SUVR). Histopathological criteria used were: CERAD [43] moderate or frequent $=$ abnormal; National Institutes of Ageing - Reagan Institute [26] Intermediate or High = abnormal; National Institutes of Ageing - Alzheimer's Association [25] Intermediate or High = abnormal; Thal 3+ Thal amyloid phase [58] of 3 or higher $=$ abnormal; Thal $4+$ Thal amyloid phase [58] of 4 or $5=$ abnormal. (DOC $241 \mathrm{~kb}$ )

Additional file 5: Clinical Status of subjects at PET Scan. Spontaneously recorded clinical notes captured at the time of PET scan. The PET category provided reflects PET majority image assessment against pathology dichotomy normal/abnormal by mCERADSOT. (DOC $155 \mathrm{~kb}$ )

Additional file 6: Amyloid plaque frequencies. In addition to the neuritic plaque frequencies assessed on Bielschowsky silver stained sections, semiquantitative plaque frequencies of none, sparse, moderate or frequent [43] were obtained from $\beta$-amyloid stained sections. In addition to the frequencies recorded in the CERAD neocortical regions, the Amyloid phase was recorded after examining multiple cortical, subcortical, midbrain and hindbrain regions [58]. (DOC $112 \mathrm{~kb}$ )

\section{Acknowledgements}

We wish to acknowledge Stacy Simpson Logan, CMPP, of Winfield Consulting, for her editorial assistance, funded by GE Healthcare and Dr Jan Wolber for critical manuscript review. This study was funded by GE Healthcare.

\section{Authors' contributions}

PS, CJB, KH, PAJ, MZ, GF, APLS designed the GE067-026 clinical trial. CJB and $\mathrm{KH}$ were responsible for oversight of blinded Vizamyl PET assessments. APLS was responsible for oversight of blinded pathology assessments. GF was Vizamyl Project Director. MDI, CAM, WEK were consulted for clinical trial design prior to initiation. AC, Jl, Al, CS, DRT were subcontracted by Covance Ltd for blinded neuropathological analyses. TGB was subcontracted by GE for blinded post-hoc striatal neuropathology analysis. MDI \& APLS prepared the manuscript. MZ, APLS performed statistical analysis. All authors approved the final manuscript.

\section{Competing interest}

The authors APLS, CB, KH, PS, PAJ, MZ and GF are full time employees of GE Healthcare. MDI, AC, Jl, Al, CS, TGB and DRT served as paid research consultants to GE Healthcare. TGB is also a paid research consultant to Avid

Radiopharmaceuticals. CAM and WEK are the co-inventors of $\left[{ }^{18} \mathrm{~F}\right]$ flutemetamol and have a conflict of interest as the result of a license agreement between the University of Pittsburgh and GE Healthcare for $\left[{ }^{18} \mathrm{~F}\right]$ flutemetamol technology.

\section{Author details}

'Department of Neurology, University of Pittsburgh, Pittsburgh, PA 15213, USA. ${ }^{2}$ GE Healthcare, The Grove Centre (GC18), White Lion Road, Amersham, Buckinghamshire HP7 9LL, UK. ${ }^{3}$ GE Healthcare, 75184 Uppsala, Sweden. ${ }^{4}$ Department of Surgical Sciences, Uppsala University, 75185 Uppsala, Sweden. ${ }^{5}$ GE Healthcare, Marlborough, MA 01752, USA. ${ }^{6}$ Department of Radiology, University of Pittsburgh, Pittsburgh, PA 15213, USA. ${ }^{7}$ Department of Psychiatry, University of Pittsburgh, Pittsburgh, PA 15213, USA. ${ }^{8}$ Pathology and Tumour Biology, Leeds Institute of Molecular Medicine, St James's University Hospital, Leeds, UK. ${ }^{9}$ National CJD Research \& Surveillance Unit University of Edinburgh Western General Hospital Edinburgh, EH4 2XU Edinburgh, UK. ${ }^{10}$ Academic Department of Neuropathology, Centre for Clinical Brain Sciences, Edinburgh EH16 4SB, UK. "'Department of Neuroscience - Laboratory for Neuropathology, KU-Leuven, Leuven, Belgium. ${ }^{12}$ Banner Sun Health Research Institute, Sun City, AZ 85351, USA. ${ }^{13}$ Geriatric Research Education and Clinical Center, VA Pittsburgh Healthcare System, Pittsburgh, USA. ${ }^{14}$ Department of Pathology, UZ Leuven, Leuven, Belgium.

Received: 16 November 2016 Accepted: 29 November 2016

Published online: 12 December 2016

\section{References}

1. Alafuzoff I, Pikkarainen M, Arzberger T, Thal DR, Al-Sarraj S, Bell J, Bodi I, Budka H, Capetillo-Zarate E, Ferrer I, Gelpi E, Gentleman S, Giaccone G, Kavantzas N, King A, Korkolopoulou P, Kovacs GG, Meyronet D, Monoranu C, Parchi P, Patsouris E, Roggendorf W, Stadelmann C, Streichenberger N, 
Tagliavini F, Kretzschmar H (2008) Inter-laboratory comparison of neuropathological assessments of beta-amyloid protein: a study of the BrainNet Europe consortium. Acta Neuropathol 115:533-546

2. Bacskai BJ, Frosch MP, Freeman SH, Raymond SB, Augustinack JC, Johnson KA, Irizarry MC, Klunk WE, Mathis CA, Dekosky ST, Greenberg SM, Hyman BT, Growdon JH (2007) Molecular imaging with Pittsburgh Compound B confirmed at autopsy: a case report. Arch Neurol 64:431-434

3. Baron JC, Farid K, Dolan E, Turc G, Marrapu ST, O'Brien E, Aigbirhio Fl, Fryer TD, Menon DK, Warburton EA, Hong YT (2014) Diagnostic utility of amyloid PET in cerebral amyloid angiopathy-related symptomatic intracerebral hemorrhage. J Cereb Blood Flow Metab 34:753-758

4. Beach TG, Monsell SE, Phillips LE, Kukull W (2012) Accuracy of the clinical diagnosis of Alzheimer disease at National Institute on Aging Alzheimer Disease Centers, 2005-2010. J Neuropathol Exp Neurol 71:266-273

5. Beach TG, Thal DR, Zanette M, Smith A, Buckley C (2016) Detection of striatal amyloid plaques with [18 F] flutemetamol: validation with postmortem histopathology. J Alzheimers Dis 52:863-873

6. Biancalana M, Koide S (2010) Molecular mechanism of Thioflavin-T binding to amyloid fibrils. Biochim Biophys Acta 1804:1405-1412

7. Braak H, Braak E (1991) Neuropathological stageing of Alzheimer-related changes. Acta Neuropathol 82:239-259

8. Braak H, Braak E (1997) Frequency of stages of Alzheimer-related lesions in different age categories. Neurobiol Aging 18:351-357

9. Burack MA, Hartlein J, Flores HP, Taylor-Reinwald L, Perlmutter JS, Cairns NJ (2010) In vivo amyloid imaging in autopsy-confirmed Parkinson disease with dementia. Neurology 74:77-84

10. Cairns NJ, Ikonomovic MD, Benzinger T, Storandt M, Fagan AM, Shah AR Reinwald LT, Carter D, Felton A, Holtzman DM, Mintun MA, Klunk WE, Morris JC (2009) Absence of Pittsburgh compound B detection of cerebral amyloid beta in a patient with clinical, cognitive, and cerebrospinal fluid markers of Alzheimer disease: a case report. Arch Neurol 66:1557-1562

11. Clark CM, Pontecorvo MJ, Beach TG, Bedell BJ, Coleman RE, Doraiswamy PM, Fleisher AS, Reiman EM, Sabbagh MN, Sadowsky CH, Schneider JA, Arora A, Carpenter AP, Flitter ML, Joshi AD, Krautkramer MJ, Lu M, Mintun MA, Skovronsky DM (2012) Cerebral PET with florbetapir compared with neuropathology at autopsy for detection of neuritic amyloid-beta plaques: a prospective cohort study. Lancet Neurol 11:669-678

12. Clark CM, Schneider JA, Bedell BJ, Beach TG, Bilker WB, Mintun MA, Pontecorvo MJ, Hefti F, Carpenter AP, Flitter ML, Krautkramer MJ, Kung HF, Coleman RE, Doraiswamy PM, Fleisher AS, Sabbagh MN, Sadowsky CH, Reiman EP, Zehntner SP, Skovronsky DM (2011) Use of florbetapir-PET for imaging beta-amyloid pathology. JAMA 305:275-283

13. Crary JF, Trojanowski JQ, Schneider JA, Abisambra JF, Abner EL, Alafuzoff I, Arnold SE, Attems J, Beach TG, Bigio EH, Cairns NJ, Dickson DW, Gearing M, Grinberg LT, Hof PR, Hyman BT, Jellinger K, Jicha GA, Kovacs GG, Knopman DS, Kofler J, Kukull WA, Mackenzie IR, Masliah E, McKee A, Montine TJ, Murray ME, Neltner JH, Santa-Maria I, Seeley WW, Serrano-Pozo A, Shelanski ML, Stein T, Takao M, Thal DR, Toledo JB, Troncoso JC, Vonsattel JP, White CL, Wisniewski T, Woltjer RL, Yamada M, Nelson PT (2014) Primary agerelated tauopathy (PART): a common pathology associated with human aging. Acta Neuropathol (Berl) 128:755-766

14. Curtis C, Gamez JE, Singh U, Sadowsky CH, Villena T, Sabbagh MN, Beach TG, Duara R, Fleisher AS, Frey KA, Walker Z, Hunjan A, Holmes C, Escovar YM, Vera CX, Agronin ME, Ross J, Bozoki A, Akinola M, Shi J, Vandenberghe R, lkonomovic MD, Sherwin PF, Grachev ID, Farrar G, Smith AP, Buckley CJ, McLain R, Salloway S (2015) Phase 3 trial of flutemetamol labeled with radioactive fluorine 18 Imaging and neuritic plaque density. JAMA Neurol 72(3):287-294

15. Dickson DW, Wertkin A, Mattiace LA, Fier E, Kress Y, Davies P, Yen SH (1990) Ubiquitin immunoelectron microscopy of dystrophic neurites in cerebellar senile plaques of Alzheimer's disease. Acta Neuropathol 79:486-493

16. Dugger BN, Serrano GE, Sue LI, Walker DG, Adler CH, Shill HA, Sabbagh MN, Caviness JN, Hidalgo J, Saxon-Labelle M, Chiarolanza G, Mariner M, HenryWatson J, Beach TG (2012) Presence of striatal amyloid plaques in parkinson's disease dementia predicts concomitant Alzheimer's disease: usefulness for amyloid imaging. J Parkinsons Dis 2:57-65

17. Effectiveness of an Electronic Training Program for Orienting and Interpreting [18 F] Flutemetamol Positron Emission Tomography (PET) Images NCT01672827. Last Updated: 12-18-2013. https://clinicaltrials.gov/ ct2/show/NCT01672827?term=NCT01672827\&rank=1 Accessed 7-20-2015.

18. Fleiss $\mathrm{JL}$ (1971) Measuring nominal scale agreement among many raters. Psychol Bull 76:378-382
19. Fodero-Tavoletti MT, Smith DP, McLean CA, Adlard PA, Barnham K, Foster LE, Leone L, Perez K, Cortes M, Culvenor JG, Li QX, Laughton KM, Rowe CC, Masters CL, Cappai R, Villemagne VL (2007) In vitro characterization of Pittsburgh compound-B binding to Lewy bodies. J Neurosci 27:10365-10371

20. Gearing M, Levey Al, Mirra SS (1997) Diffuse plaques in the striatum in Alzheimer disease (AD): relationship to the striatal mosaic and selected neuropeptide markers. J Neuropathol Exp Neurol 56:1363-1370

21. Geddes JW, Tekirian TL, Soultanian NS, Ashford JW, Davis DG, Markesbery WR (1997) Comparison of neuropathologic criteria for the diagnosis of Alzheimer's disease. Neurobiol Aging 18:S99-S105

22. Gilman S, Wenning GK, Low PA, Brooks DJ, Mathias CJ, Trojanowski JQ, Wood NW, Colosimo C, Dürr A, Fowler CJ, Kaufmann H, Klockgether T, Lees A, Poewe W, Quinn N, Revesz T, Robertson D, Sandroni P, Seppi K, Vidailhet M (2008) Second consensus statement on the diagnosis of multiple system atrophy. Neurology 71(9):670-676

23. Hauw JJ, Daniel SE, Dickson D, Horoupian DS, Jellinger K, Lantos PL, McKee A, Tabaton M, Litvan I (1994) Preliminary NINDS neuropathologic criteria for Steele-Richardson-Olszewski Syndrome (progressive Supranuclear Palsy). Neurology 44(11):2015-2019

24. Herholz K, Ebmeier K (2011) Clinical amyloid imaging in Alzheimer's disease. Lancet Neurol 10:667-670

25. Hyman BT, Phelps CH, Beach TG, Bigio EH, Cairns NJ, Carrillo MC, Dickson DW, Duyckaerts C, Frosch MP, Masliah E, Mirra SS, Nelson PT, Schneider JA, Thal DR, Thies B, Trojanowski JQ, Vinters HV, Montine TJ (2012) National Institute on Aging-Alzheimer's Association guidelines for the neuropathologic assessment of Alzheimer's disease. Alzheimers Dement 8:1-13

26. Hyman BT, Trojanowski JQ (1997) Consensus recommendations for the postmortem diagnosis of Alzheimer disease from the National Institute on Aging and the Reagan Institute Working Group on diagnostic criteria for the neuropathological assessment of Alzheimer disease. J Neuropathol Exp Neurol 56:1095-1097

27. Ikonomovic MD, Abrahamson EE, Price JC, Hamilton RL, Mathis CA, Paljug WR, Debnath ML, Cohen AD, Mizukami K, DeKosky ST, Lopez OL, Klunk WE (2012) Early AD pathology in a [C-11]PiB-negative case: a PiB-amyloid imaging, biochemical, and immunohistochemical study. Acta Neuropathol 123:433-447

28. Ikonomovic MD, Klunk WE, Abrahamson EE, Mathis CA, Price JC, Tsopelas ND, Lopresti BJ, Ziolko S, Bi W, Paljug WR, Debnath ML, Hope CE, Isanski BA, Hamilton RL, DeKosky ST (2008) Post-mortem correlates of in vivo PiB-PET amyloid imaging in a typical case of Alzheimer's disease. Brain 131:1630-1645

29. Jellinger KA, Attems J (2007) Neurofibrillary Tangle-Predominant Dementia: Comparison with Classical Alzheimer Disease. Acta Neuropathol 113(2):107-117

30. Johnson KA, Gregas M, Becker JA, Kinnecom C, Salat DH, Moran EK, Smith EE, Rosand J, Rentz DM, Klunk WE, Mathis CA, Price JC, DeKosky ST, Fischman AJ, Greenberg SM (2007) Imaging of amyloid burden and distribution in cerebral amyloid angiopathy. Ann Neurol 62:229-234

31. Johnson KA, Sperling RA, Gidicsin CM, Carmasin JS, Maye JE, Coleman RE, Reiman EM, Sabbagh MN, Sadowsky CH, Fleisher AS, Murali DP, Carpenter AP, Clark CM, Joshi AD, Lu M, Grundman M, Mintun MA, Pontecorvo MJ, Skovronsky DM (2013) Florbetapir (F18-AV-45) PET to assess amyloid burden in Alzheimer's disease dementia, mild cognitive impairment, and normal aging. Alzheimers Dement 9:S72-583

32. Kadir A, Marutle A, Gonzalez D, Scholl M, Almkvist O, Mousavi M, Mustafiz T, Darreh-Shori T, Nennesmo I, Nordberg A (2011) Positron emission tomography imaging and clinical progression in relation to molecular pathology in the first Pittsburgh Compound B positron emission tomography patient with Alzheimer's disease. Brain 134:301-317

33. Kantarci K, Yang C, Schneider JA, Senjem ML, Reyes DA, Lowe VJ, Barnes LL, Aggarwal NT, Bennett DA, Smith GE, Petersen RC, Jack CR Jr, Boeve BF (2012) Antemortem amyloid imaging and beta-amyloid pathology in a case with dementia with Lewy bodies. Neurobiol Aging 33:878-885

34. Klunk WE, Price JC, Mathis CA, Tsopelas ND, Lopresti BJ, Ziolko SK, Bi W, Hoge JA, Cohen AD, Ikonomovic MD, Saxton JA, Snitz BE, Pollen DA, Moonis M, Lippa CF, Swearer JM, Johnson KA, Rentz DM, Fischman AJ, Aizenstein HJ, DeKosky ST (2007) Amyloid deposition begins in the striatum of presenilin-1 mutation carriers from two unrelated pedigrees. J Neurosci 27:6174-6184

35. Landau SM, Breault C, Joshi AD, Pontecorvo M, Mathis CA, Jagust WJ, Mintun MA (2013) Amyloid-beta imaging with Pittsburgh compound B and florbetapir: comparing radiotracers and quantification methods. J Nucl Med $54: 70-77$ 
36. Leinonen V, Alafuzoff I, Aalto S, Suotunen T, Savolainen S, Nagren K, Tapiola T, Pirttila T, Rinne J, Jaaskelainen JE, Soininen H, Rinne JO (2008) Assessment of beta-amyloid in a frontal cortical brain biopsy specimen and by positron emission tomography with carbon 11-labeled Pittsburgh Compound B. Arch Neurol 65:1304-1309

37. Leinonen V, Rinne JO, Virtanen KA, Eskola O, Rummukainen J, Huttunen J, von Und Zu FM, Nerg O, Koivisto AM, Rinne J, Jaaskelainen JE, Buckley C, Smith A, Jones PA, Sherwin P, Farrar G, McLain R, Kailajarvi M, Heurling K, Grachev ID (2013) Positron emission tomography with [18 F] flutemetamol and $[11 \mathrm{C}] \mathrm{PiB}$ for in vivo detection of cerebral cortical amyloid in normal pressure hydrocephalus patients. Eur J Neurol 20:1043-1052

38. Leinonen V, Rinne JO, Wong DF, Wolk DA, Trojanowski JQ, Sherwin PF, Smith A, Heurling K, Su M, Grachev ID (2014) Diagnostic effectiveness of quantitative $[18 \mathrm{~F}]$ flutemetamol PET imaging for detection of fibrillar amyloid beta using cortical biopsy histopathology as the standard of truth in subjects with idiopathic normal pressure hydrocephalus. Acta Neuropathol Commun 2:46

39. Litchfield S, Nagy Z (2001) New temperature modification makes the Bielschowsky silver stain reproducible. Acta Neuropathol 101:17-21

40. Litvan I, Hauw JJ, Bartko JJ, Lantos PL, Daniel SE, Horoupian DS, McKee A, Dickson D, Bancher C, Tabaton M, Jellinger K, Anderson DW (1996) Validity and reliability of the preliminary NINDS neuropathologic criteria for progressive supranuclear palsy and related disorders. J Neuropathol Exp Neurol 55(1):97-105

41. Lockhart A, Ye L, Judd DB, Merritt AT, Lowe PN, Morgenstern JL, Hong G, Gee AD, Brown J (2005) Evidence for the presence of three distinct binding sites for the thioflavin T class of Alzheimer's disease PET imaging agents on beta-amyloid peptide fibrils. J Biol Chem 280:7677-7684

42. McKeith IG, Ballard CG, Perry RH, Ince PG, O'Brien JT, Neill D, Lowery K, Jaros E, Barber R, Thompson P, Swann A, Fairbairn AF, Perry EK (2000) Prospective validation of consensus criteria for the diagnosis of dementia with lewy bodies. Neurology 54(5):1050-1058

43. Mirra SS, Heyman A, McKeel D, Sumi SM, Crain BJ, Brownlee LM, Vogel FS, Hughes JP, van Belle G, Berg L (1991) The Consortium to Establish a Registry for Alzheimer's Disease (CERAD). Part II. Standardization of the neuropathologic assessment of Alzheimer's disease. Neurology 41:479-486

44. Moossy J, Zubenko GS, Martinez AJ, Rao GR (1988) Bilateral symmetry of morphologic lesions in Alzheimer's disease. Arch Neurol 45:251-254

45. Murray ME, Lowe VJ, Graff-Radford NR, Liesinger AM, Cannon A, Przybelski SA, Rawal B, Parisi JE, Petersen RC, Kantarci K, Ross OA, Duara R, Knopman DS, Jack CR Jr, Dickson DW (2015) Clinicopathologic and 11C-Pittsburgh compound B implications of Thal amyloid phase across the Alzheimer's disease spectrum. Brain 138:1370-1381

46. National Electrical Manufacturers Association and The International Electrotechnical Commision NEMA IEC Body Phantom Set. Last Updated: 2007. http://www.spect.com/pub/NEMA_IEC_Body_Phantom_Set.pdf Accessed 9-18-2015.

47. Nelissen N, Van LK, Thurfjell L, Owenius R, Vandenbulcke M, Koole M, Bormans G, Brooks DJ, Vandenberghe R (2009) Phase 1 study of the Pittsburgh compound B derivative 18 F-flutemetamol in healthy volunteers and patients with probable Alzheimer disease. J Nucl Med 50:1251-1259

48. Raji CA, Becker JT, Tsopelas ND, Price JC, Mathis CA, Saxton JA, Lopresti BJ, Hoge JA, Ziolko SK, DeKosky ST, Klunk WE (2008) Characterizing regional correlation, laterality and symmetry of amyloid deposition in mild cognitive impairment and Alzheimer's disease with Pittsburgh Compound B. J Neurosci Methods 172:277-282

49. Re-Read Study to Compare the Brain Uptake of [18 F] Flutemetamol With Brain Neuritic Plaque Density Determined Postmortem NCT02090855. Last Updated: 7-11-2014. https://clinicaltrials.gov/ct2/show/NCT02090855?term= NCT02090855\&rank=1 Accessed 7-20-2015.

50. Rinne JO, Frantzen J, Leinonen V, Lonnrot K, Laakso A, Virtanen KA, Solin O, Kotkansalo A, Koivisto A, Sajanti J, Karppinen A, Lehto H, Rummukainen J, Buckley C, Smith A, Jones PA, Sherwin P, Farrar G, McLain R, Kailajarvi M, Grachev ID (2013) Prospective flutemetamol positron emission tomography and histopathology in normal pressure hydrocephalus. Neurodegener Dis 13:237-245

51. Rinne JO, Wong DF, Wolk DA, Leinonen V, Arnold SE, Buckley C, Smith A, McLain R, Sherwin PF, Farrar G, Kailajarvi M, Grachev ID (2012) [(18)F] Flutemetamol PET imaging and cortical biopsy histopathology for fibrillar amyloid beta detection in living subjects with normal pressure hydrocephalus: pooled analysis of four studies. Acta Neuropathol 124:833-845

52. Rosenberg PB, Wong DF, Edell SL, Ross JS, Joshi AD, Brasic JR, Zhou Y, Raymont V, Kumar A, Ravert HT, Dannals RF, Pontecorvo MJ, Skovronsky
DM, Lyketsos CG (2013) Cognition and amyloid load in Alzheimer disease imaged with florbetapir F 18(AV-45) positron emission tomography. Am J Geriatr Psychiatry 21:272-278

53. Sojkova J, Driscoll I, lacono D, Zhou Y, Codispoti KE, Kraut MA, Ferrucci L, Pletnikova O, Mathis CA, Klunk WE, O'Brien RJ, Wong DF, Troncoso JC, Resnick SM (2011) In vivo fibrillar beta-amyloid detected using [11C]PiB positron emission tomography and neuropathologic assessment in older adults. Arch Neurol 68:232-240

54. Summary of Product Characteristics, Vizamyl. Last Updated: 10-16-2014. http://www.ema.europa.eu/docs/en_GB/document_library/EPAR_-_Product_ Information/human/002557/WC500172950.pdf Accessed 3-2-2015.

55. Thal DR, Beach TG, Zanette M, Heurling K, Chakrabarty A, Ismail A, Smith AP, Buckley C (2015) [(18)F] flutemetamol amyloid positron emission tomography in preclinical and symptomatic Alzheimer's disease: Specific detection of advanced phases of amyloid-beta pathology. Alzheimers Dement 11:975-985

56. Thal DR, Ghebremedhin E, Orantes M, Wiestler OD (2003) Vascular pathology in Alzheimer disease: correlation of cerebral amyloid angiopathy and arteriosclerosis/lipohyalinosis with cognitive decline. J Neuropathol Exp Neurol 62:1287-1301

57. Thal DR, Ghebremedhin E, Rub U, Yamaguchi H, Del TK, Braak H (2002) Two types of sporadic cerebral amyloid angiopathy. J Neuropathol Exp Neurol 61:282-293

58. Thal DR, Rub U, Orantes M, Braak H (2002) Phases of A beta-deposition in the human brain and its relevance for the development of AD. Neurology 58:1791-1800

59. Thurfjell L, Lilja J, Lundqvist R, Buckley C, Smith A, Vandenberghe R, Sherwin P (2014) Automated quantification of 18 F-flutemetamol PET activity for categorizing scans as negative or positive for brain amyloid: concordance with visual image reads. J Nucl Med 55:1623-1628

60. Vemuri P, Whitwell JL, Kantarci K, Josephs KA, Parisi JE, Shiung MS, Knopman DS, Boeve BF, Petersen RC, Dickson DW, Jack CR Jr (2008) Antemortem MRI based STructural Abnormality iNDex (STAND)-scores correlate with postmortem Braak neurofibrillary tangle stage. Neuroimage 42:559-567

61. Villemagne VL, Klunk WE, Mathis CA, Rowe CC, Brooks DJ, Hyman BT, Ikonomovic MD, Ishii K, Jack CR, Jagust WJ, Johnson KA, Koeppe RA, Lowe VJ, Masters CL, Montine TJ, Morris JC, Nordberg A, Petersen RC, Reiman EM, Selkoe DJ, Sperling RA, Van LK, Weiner MW, Drzezga A (2012) Abeta Imaging: feasible, pertinent, and vital to progress in Alzheimer's disease. Eur J Nucl Med Mol Imaging 39:209-219

62. Villemagne $\mathrm{V}$, McLean CA, Reardon K, Boyd A, Lewis V, Klug G, Jones G, Baxendale D, Masters CL, Rowe CC, Collins SJ (2009) 11C-PiB PET studies in typical sporadic Creutzfeldt-Jakob disease. J Neurol Neurosurg Psychiatry 80:998-1001

63. Vizamy $\left.\right|^{\mathrm{TM}}$ (flutemetamol F 18 injection) Electronic Reader Training Program. Last Updated: 2014. http://www.readvizamyl.com Accessed 8-4-2014.

64. Vonsattel JP, Myers RH, Hedley-Whyte ET, Ropper AH, Bird ED, Richardson EP Jr (1991) Cerebral amyloid angiopathy without and with cerebral hemorrhages: a comparative histological study. Ann Neurol 30:637-649

65. Wolk DA, Grachev ID, Buckley C, Kazi H, Grady MS, Trojanowski JQ, Hamilton RH, Sherwin P, McLain R, Arnold SE (2011) Association between in vivo fluorine 18-labeled flutemetamol amyloid positron emission tomography imaging and in vivo cerebral cortical histopathology. Arch Neurol 68:1398-1403

66. Wolk DA, Zhang Z, Boudhar S, Clark CM, Pontecorvo MJ, Arnold SE (2012) Amyloid imaging in Alzheimer's disease: comparison of florbetapir and Pittsburgh compound-B positron emission tomography. J Neurol Neurosurg Psychiatry 83:923-926

67. Wong DF, Moghekar AR, Rigamonti D, Brasic JR, Rousset O, Willis W, Buckley C, Smith A, Gok B, Sherwin P, Grachev ID (2013) An in vivo evaluation of cerebral cortical amyloid with [18 F] flutemetamol using positron emission tomography compared with parietal biopsy samples in living normal pressure hydrocephalus patients. Mol Imaging Biol 15:230-237

68. Yamada M (2003) Senile Dementia of the Neurofibrillary Tangle Type (tangle-Only Dementia): Neuropathological Criteria and Clinical Guidelines for Diagnosis. Neuropathology 23(4):311-317

69. Yamaguchi H, Nakazato Y, Hirai S, Shoji M, Harigaya Y (1989) Electron micrograph of diffuse plaques. Initial stage of senile plaque formation in the Alzheimer brain. Am J Pathol 135:593-597

70. Ye L, Velasco A, Fraser G, Beach TG, Sue L, Osredkar T, Libri V, Spillantini MG, Goedert M, Lockhart A (2008) In vitro high affinity alpha-synuclein binding sites for the amyloid imaging agent PIB are not matched by binding to Lewy bodies in postmortem human brain. J Neurochem 105:1428-1437 